\title{
INVOLUTIONS AND COMMUTATORS IN ORTHOGONAL GROUPS
}

\author{
FRIEDER KNÜPPEL and GERD THOMSEN
}

(Received 8 August 1996; revised 3 October 1997)

Communicated by R. Howlett

\begin{abstract}
Suppose we are given a regular symmetric bilinear form on a finite-dimensional vector space $V$ over a commutative field $K$ of characteristic $\neq 2$. We want to write given elements of the commutator subgroup $\Omega(V)$ (of the orthogonal group $O(V)$ ) and also of the kernel of the spinorial norm $\operatorname{ker}(\Theta)$ as (short) products of involutions and as products of commutators.
\end{abstract}

1991 Mathematics subject classification (Amer. Math. Soc.): primary 11 E57 secondary 14L35.

\section{Introduction}

Let $V$ be an $n$-dimensional vector space ( $n$ finite) over a commutative field $K$ of characteristic distinct from 2 . Let $f: V \times V \rightarrow K$ be a regular symmetric bilinear form.

Whenever $G$ is a group and $S$ a generating set for $G$ with $S^{-1}=S$ we are challenged to find for each $g \in G$ the length $\mathrm{l}_{S}(g):=\min \left\{k \in \mathbb{N}_{0} \mid g\right.$ is a product of $k$ elements of $S\}$ and also the global length $l_{S}(G):=\max \left\{l_{S}(g) \mid g \in G\right\} \in \mathbb{N} \cup\{\infty\}$. If $S$ is the set of all involutions in $\mathrm{G}$ and $\mathrm{l}_{S}(G) \leq m$ then $G$ is called $m$-reflectional.

We study the length-problem within the following framework:

(1) $G=\Omega(V)$ is the commutator subgroup of the orthogonal group $\mathrm{O}(V)$ and

(la) $S$ is the set of all involutions of $G$, or

(1b) $S$ is the set of all commutators in elements of $\mathrm{O}(V)$, or

(1c) $S$ is the set of all commutators in elements of $G=\Omega(V)$.

(2) $G=\operatorname{ker}(\Theta)$ is the kernel of the spinorial norm on $\mathrm{O}(V)$, and

(C) 1998 Australian Mathematical Society $0263-6115 / 98 \$ A 2.00+0.00$ 
(2a) $S$ is the set of all involutions in $G$, or

(2b) $S$ is the set of all symmetries in $G$.

It is well known that $\mathrm{O}(V)$ is 2-reflectional. Our approach is based on a careful analysis of the possible choices of involutions $\rho, \sigma \in \mathrm{O}(V)$ with the property $\pi=\rho \sigma$ for a given $\pi \in O(V)$. The results in Section 5 are of interest in their own right and yield facts on normal forms of orthogonal mappings.

As to (1a), we can prove under appropriate assumptions on the underlying field $K$ that $\Omega(V)$ is 3-reflectional. Our results cover in particular finite fields and euclidean fields; if $K$ is finite we find out precisely all cases when $\Omega(V)$ is 2-reflectional. Analogue theorems are obtained for $G=\operatorname{ker}(\Theta)$, problem (2a). Results are Theorem 7.5, Corollary 7.6, Lemma 8.3 and Theorems $8.5,8.6,8.8$.

Problem (2b) is essentially covered by [9]; this article solves the following task: write a given $\pi \in \mathrm{O}(V)$ as a product of (as few as possible) symmetries out of given conjugacy classes in $\mathrm{O}(V)$. We obtain Corollary 6.2.

Let $G$ be a group. A commutator (in elements of $G$ ) is an element of the form $\alpha \beta \alpha^{-1} \beta^{-1}$ where $\alpha, \beta \in G$. The subgroup generated by the set of all commutators is the commutator subgroup $G^{\prime}$ of $G$. Each $\pi \in G^{\prime}$ is a product of commutators; let $\mathrm{cl}_{G}(\pi)$ denote the minimal number of factors in such a product, and $\operatorname{cl}(G):=$ $\max \left\{\operatorname{cl}_{G}(\pi) \mid \pi \in G^{\prime}\right\} \in \mathbb{N} \cup\{\infty\}$. O. Ore conjectured that every element of a finite simple non-abelian group is a commutator. This was proved for the alternating group $\mathrm{A}_{n}$ (where $n \geq 5$ ) in [7] and also in [14]. In [17] Thompson proved that each element of $\operatorname{PSL}(V)$ is a commutator in elements of $\operatorname{PSL}(V)$, provided $\operatorname{dim}(V) \geq 3$ or $|K| \geq 4$. Nielsen (cf. [13]) proved that in a symplectic group $\mathrm{Sp}(V)$ one can always find a conjugacy class $\Sigma$ such that $\operatorname{Sp}(V)=\Sigma^{2} \cup\{-1\}$. Hence, in a projective symplectic group $\operatorname{PSp}(V)$ one can always find a conjugacy class $\Sigma$ such that $\operatorname{PSp}(V)=\Sigma^{2}$. In particular, every element of $\operatorname{PSp}(V)$ is a commutator. Now let $G=\Omega(V)$ be the commutator subgroup of $\mathrm{O}(V)$. If the field is algebraically closed it is known that $\operatorname{cl}(\Omega(V))=1$; cf. [15]. Our main results cover fields with the u-invariant (defined in Section 2$) \mathrm{u}(K) \leq 2$ (this is valid for each finite field) and $\mathbb{R}$. Then we obtain that each element of $\Omega(V)$ is a product of two conjugate orthogonal involutions, hence a commutator in elements of $\mathrm{O}(V)$. Furthermore, each element of $\Omega(V)$ is a product of 2 commutators in elements of $\Omega(V)$. If $K=\mathbb{R}$ and the Witt-index satisfies ind $(V) \leq 1$ then each element of $\Omega(V)$ is a commutator in elements of $\Omega(V)$. Main results are Corollary 9.5 and Theorems 9.6, 9.8, 9.9.

A. J. Hahn studies a closely related problem in [2]. The set of commutators of symmetries generates $\Omega(V)$ and the associated length problem is solved under the assumption that $K$ is a non-dyadic field.

Most of the results of this paper are from Thomsen's dissertation [18]. 


\section{Basic definitions and facts}

The definitions and facts compiled in the following two sections will be used without a particular reference.

Let $V$ be an $n$-dimensional vector space ( $n$ finite) over a commutative field $K$ of characteristic distinct from 2. Let $f: V \times V \rightarrow K$ be a regular symmetric bilinear form.

We write $q(v):=f(v, v)$. Let $\mathrm{O}^{+}(V):=\{\pi \in \mathrm{O}(V) \mid \operatorname{det}(\pi)=1\}$ denote the special orthogonal group and $\mathrm{O}^{-}(V):=\{\pi \in \mathrm{O}(V) \mid \operatorname{det}(\pi)=-1\}$. For a subspace $U$ of $V$ let $\mathrm{d} U:=\operatorname{det}(G r) K^{* 2}$ denote the discriminant of $U$ where $G r$ is an arbitrary Gram-matrix of the form $\left.f\right|_{U \times U}$. The Witt-index ind $(V)$ is the number of hyperbolic planes in a Witt-decomposition of $V$.

For any field $K$ define the u-invariant $\mathrm{u}(K):=\max \left\{k \in \mathbb{N} \cup\{\infty\} \mid K^{k}\right.$ admits a symmetric anisotropic bilinear form\}.

A vector space $V$ with a symmetric bilinear form $f$ is called universal if $\{f(v, v) \mid$ $v \in V\}=K$.

REMARK 2.1. If $\mathrm{u}(K)<\infty$ then $\mathrm{u}(K)=\min \{k \in \mathbb{N} \mid$ each $k$-dimensional regular $K$-vector space is universal $\}$.

A field $K$ is called (formally) real if -1 is not a sum of squares. If $K$ is not formally real then call $\mathrm{s}(K):=\min \{k \in \mathbb{N} \mid-1$ is a sum of $k$ squares $\}$ the level of $K$.

A field $K$ is called a euclidean field if $K$ is formally real and $K^{*}$ consists of precisely two classes of squares, that is, $K^{*}=K^{* 2} \cup-K^{* 2}$. So $K^{* 2}$ is the only positive-domain making $K$ an ordered field.

We compile some well-known facts.

LEMMA 2.2. (a) If $K$ is a non formally real field then $\mathrm{s}(K) \leq \mathrm{u}(K) \leq\left|K^{*} / K^{* 2}\right|$. (b) If $K$ is finite (with $\operatorname{char}(K) \neq 2$ ) then $\mathrm{u}(K)=\left|K^{*} / K^{* 2}\right|=2$. If $K$ is algebraically closed then $u(K)=1$.

If $K$ is a euclidean field then there is a number $r \in \mathbb{N}_{0}$ such that each orthogonal basis for $V$ contains $r$ vectors $v$ with $f(v, v)>0$ and $s=n-r$ vectors with $f(v, v)<0$. Call $\operatorname{sgn}(V):=(r, s)$ the signature of $V$.

\section{LEMMA 2.3. Suppose that $U$ and $W$ are regular $K$-vector spaces.}

(a) If $\mathrm{u}(K) \leq 2$ then $U$ is isometric to $W$ if and only if $\operatorname{dim}(U)=\operatorname{dim}(W)$ and $\mathrm{d} U=\mathrm{d} W$.

(b) If $K$ is a euclidean field then $U$ is isometric to $W$ if and only if $\operatorname{sgn}(U)=\operatorname{sgn}(W)$. 
DEFINITION 2.4 (basic concepts and observations). (a) For a linear mapping $\pi$ : $V \rightarrow V$ and $j \in \mathbb{N}$ let $\mathrm{B}^{j}(\pi):=V(\pi-1)^{j}$ and $\mathrm{F}^{j}(\pi):=\operatorname{ker}\left((\pi-1)^{j}\right)$. We call $\mathrm{B}(\pi):=\mathrm{B}^{1}(\pi)$ the path (some authors use the term residue-space) and $\mathrm{F}(\pi):=$ $\mathrm{F}^{1}(\pi)$ the fixed space of $\pi$. Furthermore, let $\mathrm{B}^{\infty}(\pi):=\bigcap\left\{\mathrm{B}^{j}(\pi) \mid j \in \mathbb{N}\right\}$ and $\mathrm{F}^{\infty}(\pi):=\bigcup\left\{\mathrm{F}^{j}(\pi) \mid j \in \mathbb{N}\right\}$.

(b) Let $\pi \in \mathrm{GL}(V)$. Then the negative-space satisfies $\mathrm{N}(\pi):=\mathrm{F}(-\pi) \subseteq \mathrm{B}(\pi)$. Furthermore, $\pi^{2}=1$ if and only if $\mathrm{N}(\pi)=\mathrm{B}(\pi)$.

(c) If $\pi \in \mathrm{O}(V)$ then $\mathrm{B}(\pi)^{\perp}=\mathrm{F}(\pi)$; in particular, $\operatorname{rad}(\mathrm{B}(\pi))=\mathrm{B}(\pi) \cap \mathrm{F}(\pi)$. Furthermore, $V=\mathrm{B}^{\infty}(\pi) \oplus \mathrm{F}^{\infty}(\pi)$.

(d) Call $\pi \in \mathrm{O}(V)$ regular (isotropic, anisotropic and so on) if $\mathrm{B}(\pi)$ has this property. If $\pi$ is regular then $V=\mathrm{B}(\pi) \oplus \mathrm{F}(\pi)$.

(e) Let $\pi \in \mathrm{O}(V)$. Then $\pi \in \mathrm{O}^{+}(V)$ if and only if $\operatorname{dim}(\mathrm{B}(\pi))$ is even.

If $\sigma \in \mathrm{O}(V)$ is simple, that is, $\operatorname{dim}(\mathrm{B}(\sigma))=1$, then $\sigma$ is a symmetry (that is, $\sigma^{2}=1$ and $\left.\operatorname{dim}(\mathrm{B}(\sigma))=1\right)$.

(f) Let $W \leq V$ be a subspace of $V$. Then $f$ induces a regular symmetric bilinear form on $W / \operatorname{rad}(W)$.

(g) The spinorial norm $\Theta: \mathrm{O}(V) \rightarrow K^{*} / K^{* 2}$ is the homomorphism with the property $\Theta\left(\sigma_{a}\right)=\mathrm{d}\langle a\rangle$ where $a$ is any anisotropic vector and $\sigma_{a}$ denotes the symmetry whose negative space is $\langle a\rangle$.

REMARK 2.5 (invariant subspaces). Let $\pi \in \mathrm{GL}(V)$ and $U \leq V$ such that $\mathrm{B}(\pi) \leq$ $U$. Then $U \pi=U$.

LEMMA 2.6 (path-lemma). Let $\pi$ and $\sigma_{i}$ be linear mappings of $V$ such that $\pi=$ $\sigma_{1} \cdots \sigma_{k}$. Then $\mathrm{B}(\pi) \leq \mathrm{B}\left(\sigma_{1}\right)+\cdots+\mathrm{B}\left(\sigma_{k}\right)$.

LEMMA 2.7. Let $\pi=\rho \sigma$ where $\rho, \sigma \in \mathrm{GL}(V)$ are involutions. Then

$$
\begin{aligned}
|\operatorname{dim}(\mathrm{B}(\rho))-\operatorname{dim}(\mathrm{B}(\sigma))| & \leq \operatorname{dim}(\mathrm{N}(\pi)) \quad \text { and } \\
|\operatorname{dim}(\mathrm{B}(\rho))-\operatorname{dim}(\mathrm{F}(\sigma))| & \leq \operatorname{dim}(\mathrm{F}(\pi))
\end{aligned}
$$

(a) Suppose additionally that $\mathrm{F}(\pi)=0=\mathrm{N}(\pi)$. Then $n$ is even and $\operatorname{dim}(\mathrm{B}(\rho))=$ $n / 2=\operatorname{dim}(B(\sigma))$.

(b) Suppose additionally that $\mathrm{F}(\pi)=0$ and $\operatorname{dim}(\mathrm{N}(\pi))=1$. If $n$ is even then $\operatorname{dim}(\mathrm{B}(\rho))=n / 2=\operatorname{dim}(\mathrm{B}(\sigma))$. If $n$ is odd then $\operatorname{dim}(\mathrm{B}(\rho))=(n+1) / 2$ and $\operatorname{dim}(\mathrm{B}(\sigma))=(n-1) / 2$, or $\operatorname{dim}(\mathrm{B}(\rho)=(n-1) / 2$ and $\operatorname{dim}(\mathrm{B}(\sigma))=(n+1) / 2$.

(c) Suppose additionally that $\mathrm{N}(\pi)=0$ and $\operatorname{dim}(\mathrm{F}(\pi))=1$. If $n$ is even then $\operatorname{dim}(\mathrm{B}(\rho))=n / 2=\operatorname{dim}(\mathrm{B}(\sigma))$. If $n$ is odd then $\operatorname{dim}(\mathrm{B}(\rho))=(n+1) / 2=$ $\operatorname{dim}(\mathrm{B}(\sigma))$, or $\operatorname{dim}(\mathrm{B}(\rho))=(n-1) / 2=\operatorname{dim}(\mathrm{B}(\sigma))$. 
PROOF. Clearly $(\mathrm{B}(\sigma) \cap \mathrm{F}(\rho)) \oplus(\mathrm{B}(\rho) \cap \mathrm{F}(\sigma)) \leq \mathrm{N}(\pi)$ and $(\mathrm{B}(\sigma) \cap \mathrm{B}(\rho)) \oplus$ $(\mathrm{F}(\rho) \cap \mathrm{F}(\sigma)) \leq \mathrm{F}(\pi)$. The assertions are almost immediate conclusions.

LEMMA 2.8. Let $\alpha, \beta \in \mathrm{O}(V, f)$. If $\mathrm{B}(\alpha) \cap \mathrm{B}(\beta)=\{0\}$ then $\mathrm{B}(\alpha \beta)=\mathrm{B}(\alpha) \oplus \mathrm{B}(\beta)$.

PROOF. Any two linear mappings $\alpha, \beta: V \rightarrow V$ satisfy $\operatorname{dim}(\mathrm{F}(\alpha \beta)) \leq \operatorname{dim}(\mathrm{F}(\alpha) \cap$ $\mathrm{F}(\beta))+\operatorname{dim}(\mathrm{B}(\alpha) \cap \mathrm{B}(\beta))$. Hence our assumptions yield $\operatorname{dim}(\mathrm{F}(\alpha \beta)) \leq \operatorname{dim}(\mathrm{F}(\alpha) \cap$ $\mathrm{F}(\beta))$. Taking orthogonal spaces finishes the proof.

DEFINITION 2.9. For a polynomial $q=\sum a_{j} x^{j}$ with $a_{0} \neq 0$ and degree $m$ let $q^{*}:=a_{0}^{-1} \sum a_{m-j} x^{j}$ denote the reciprocal polynomial.

If $q$ is the minimum (characteristic) polynomial of $\pi \in \mathrm{GL}(V)$ (denotion: $\operatorname{mip}(\pi)$ respectively $\operatorname{char}(\pi)$ ) then $q^{*}$ is the minimum (characteristic) polynomial of $\pi^{-1}$. In particular, as $\pi \in \mathrm{O}(V)$ is conjugate to its inverse (in $\mathrm{GL}(V)$ ), the minimum and also the characteristic polynomial of $\pi \in \mathrm{O}(V)$ is symmetric (that is, $q=q^{*}$ ).

Lemma 2.10. Let $\pi \in \mathrm{O}(V)$ and $\operatorname{mip}(\pi)=p^{k}$ where $p \in K[x]$. Let $j \in \mathbb{N}$, $j \leq k$. Then $\operatorname{ker}\left(p^{j}(\pi)\right) \perp V p^{j}(\pi)$. In particular, if $j \leq k / 2$ and $V$ is $\pi$-cyclic then $\operatorname{ker}\left(p^{j}(\pi)\right)$ is totally isotropic.

PROOF. Let $v \in \operatorname{ker}\left(p^{j}(\pi)\right)$ and $y \in V p^{j}(\pi)$. Then $y=z p^{j}(\pi)$ for some $z \in V$. As $p$ is symmetric (as we observed in the above) we obtain $f(y, v)=f\left(z p^{j}(\pi), v\right)=$ $f\left(z, p^{j}(0) \cdot v p^{j}(\pi) \cdot \pi^{-j \cdot d \operatorname{degree}(p)}\right)=f(z, 0)=0$. If $V$ is $\pi$-cyclic then $V p^{j}(\pi)=$ $\operatorname{ker}\left(p^{k-j}(\pi)\right)$ and the last assertion follows.

\section{Orthogonal decompositions}

LEMMA 3.1. Let $\pi \in \mathrm{O}(V), g, h \in K[x]$ and $g^{*}$ prime to $h$. Then $\operatorname{ker}(g(\pi)) \perp$ $\operatorname{ker}\left(h(\pi)\right.$. Special case: If $g$ is prime to $g^{*}$ then $\operatorname{ker}(g(\pi))$ is totally isotropic.

We recall the following facts on orthogonal normal-forms which can be found in [6].

DEFINITION 3.2. Let $\pi \in \mathrm{O}(V, f)$. Call $V$ an orthogonally indecomposable $\pi$ module if $V=U(1) W$ for $\pi$-modules $U$ and $W$ implies that $U=\{0\}$ or $W=\{0\}$.

REMARK 3.3. Let $\pi \in \mathrm{GL}(V)$. Then $V$ is called an indecomposable $\pi$-module if a proper decomposition $V=U \oplus W$ into $\pi$-modules $U$ and $W$ does not exist. Recall 
that $V$ is an indecomposable $\pi$-module if and only if $V$ is a $\pi$-cyclic module whose minimum polynomial (=characteristic polynomial as $V$ is $\pi$-cyclic) is the power of an irreducible polynomial. Distinguish carefully the concepts 'indecomposable' and 'orthogonally indecomposable'.

LEMMA 3.4 (orthogonal decomposition into $\pi$-modules). Let $\pi \in \mathrm{O}(V, f)$. Then $V$ admits a decomposition $V=V_{1} \oplus \cdots(1) V_{k}$ into orthogonally indecomposable $\pi$-modules $V_{i}$. In any two such decompositions the numbers of orthogonally indecomposable $\pi$-modules with the same given minimum polynomial are equal.

The structure of orthogonally indecomposable $\pi$-modules is well-known and has been described by various authors, for example, [6].

Definition 3.5 (types). Let $\pi \in \mathrm{O}(V)$. We say $\pi$-type $(V)=\Delta$ if $V$ is an orthogonally indecomposable $\pi$-module and $\Delta$ is explained as follows.

$\pi-\operatorname{type}(V)=1$ (more precisely: $1^{+}, 1^{-}$): This means that $V=U \oplus W$ where $U, W$ are totally isotropic indecomposable $\pi$-modules such that $\operatorname{mip}\left(\pi_{U}\right)=(x-1)^{2 t}=$ $\operatorname{mip}\left(\pi_{W}\right)$ respectively $\operatorname{mip}\left(\pi_{U}\right)=(x+1)^{2 t}=\operatorname{mip}\left(\pi_{W}\right)$ (hence $\left.n=4 t\right)$.

$\pi-\operatorname{type}(V)=2$ (more precisely: $2^{*}, 2^{-}, 2^{+}$): This means that $V$ is an indecomposable $\pi$-module, say mip $(\pi)=p^{t}$ where $p$ is an irreducible polynomial. In particular, $V$ is a $\pi$-cyclic module. If $p \neq x-1, x+1$ then $\mathrm{p}$ is symmetric and has even degree and we write $\pi$-type $(V)=2^{*}$. If $p=x-1$, respectively $p=x+1$, then $t$ is odd and we use the notation $\pi$-type $(V)=2^{-}$, respectively $\pi$-type $(V)=2^{+}$.

$\pi-\operatorname{type}(V)=3$ : This means that $V=U \oplus W$ where $U$ and $W$ are indecomposable $\pi$-modules whose minimum polynomials are $p^{t}$, respectively $p^{* t}$, and $p$ is an irreducible polynomial where $p$ is prime to $p^{*}$. This implies that $U$ and $W$ are totally isotropic and that $V$ is a $\pi$-cyclic module.

THEOREM 3.6 (type-classification). Each orthogonally indecomposable $\pi$-module $\neq 0$ fits into precisely one of the three types given above.

\section{Some basic tools}

We collect some well-known facts. Proofs can be found, for example, in [3].

LEMMA 4.1. Let ind $(V) \geq 1$. Then

(a) $\Omega(V)=O^{+}(V) \cap \operatorname{ker}(\Theta)$.

(b) If $n \geq 5$ then $P \Omega(V)$ is simple. 
LEMMA 4.2. (a) If $n=3$ and ind $(V)=1$ then $\Omega(V) \cong P S L_{2}(K)$.

(b) If $n=4$ and ind $(V)=1$ then $\Omega(V) \cong P S L_{2}(K(\delta))$ where $\delta^{2} K^{* 2}=\mathrm{d} V$.

(c) If $n=4$ and ind $(V)=2$ then $P \Omega(V) \cong P S L_{2}(K) \times P S L_{2}(K)$.

LEMMA 4.3. (a) Let $\sigma \in \mathrm{O}(V)$ be an involution. Then $\Theta(\sigma)=\mathrm{dB}(\sigma)$.

(b) (Zassenhaus-formula) $\Theta(\pi)=\operatorname{det}\left(\left.\frac{1}{2}(1-\pi)\right|_{\mathrm{B}^{\infty}(\pi)}\right) \cdot \mathrm{dB}^{\infty}(\pi)$ for each $\pi \in \mathrm{O}(V)$. In particular, $\pi \in \operatorname{ker}(\Theta)$ when $\pi$ is unipotent.

LEMMA 4.4. Let $V=U \oplus W$ where $U$ and $W$ are totally isotropic. Then every $\alpha \in$ $\mathrm{GL}(U)$ admits a unique $\varphi \in \mathrm{O}(V)$ such that $\left.\varphi\right|_{U}=\alpha$ and $W \varphi=W$. Furthermore, $\varphi \in O^{+}(V)($ as $\operatorname{dim}(\mathrm{B}(\varphi))$ is even). If $\alpha$ is an involution then $\varphi$ is also an involution.

PROOF. This follows from a simple matrix calculation.

Next, we state a theorem which is due to [21]; however, this proof contains a gap. For the real and the complex numbers Frobenius gave a proof in 1910.

\section{PROPOSITION 4.5. The orthogonal group $\mathrm{O}(V)$ is 2-reflectional.}

We outline a short proof. Given $\pi \in O(V)$. We want to write $\pi$ as a product of two orthogonal involutions. Hence we can assume that $V$ is an orthogonally indecomposable $\pi$-module. If $\pi$-type $(V) \in\{2,3\}$ then $V$ is a $\pi$-cyclic module; that is, we find $v \in V$ such that $v, v \pi, \ldots, v \pi^{n-1}$ is a basis for $V$. Define a linear mapping $\rho: V \rightarrow V, v \pi^{j} \mapsto v \pi^{n-1-j}$ for $j \in\{0, \ldots, n-1\}$. Clearly, $\rho$ is an involution, and it is easy to check that $\rho \in \mathrm{O}(V)$. From the fact that $\operatorname{mip}(\pi)$ is symmetric it follows that $\sigma:=\rho \pi$ is an involution. Now suppose that $\pi-\operatorname{type}(V)=1$. Let $U$ and $W$ denote the cyclic $\pi$-modules occurring in Lemma 3.5. We define involutions $\rho_{U}, \sigma_{U} \in \mathrm{GL}(U)$ such that $\pi_{U}=\rho_{U} \sigma_{U}$ by the same definition as above (with $U$ instead of $V$ ). Now Lemma 4.4 implies that $\rho_{U}$ and $\sigma_{U}$ admit (unique) liftings to orthogonal involutions $\rho, \sigma \in \mathrm{O}(V)$ that leave $W$ invariant. The uniqueness statement of Lemma 4.4 yields that $\pi=\rho \sigma$.

The following proposition was proved in [10].

PROPOSITION 4.6 (involutions-invariance-theorem). If $\pi=\rho \sigma$ where $\rho, \sigma \in$ $\mathrm{O}(V)$ are involutions then $V$ admits an orthogonal decomposition into orthogonally indecomposable $\pi$-modules that are simultaneously $\rho$-modules and $\sigma$-modules.

LEMMA 4.7 (discriminant, spinorial norm of orthogonally indecomposable modules). Let $\pi \in \mathrm{O}(V), p:=\operatorname{mip}(\pi)$ and $V$ an orthogonally indecomposable $\pi$ module. 
(a) If $\pi-\operatorname{type}(V)=2^{*}$ or 3 then

$$
\mathrm{d} V=p(1) p(-1) K^{* 2} \text { and } \Theta(\pi)=p(-1) K^{* 2} .
$$

(b) If $\pi$-type $(V)=3$ where $p=q q^{*}$ then $V=U \oplus W$ where $U:=\operatorname{ker}(q(\pi))$ and $W:=\operatorname{ker}\left(q^{*}(\pi)\right.$ are indecomposable $\pi$-modules and

$$
\Theta(\pi)=(-1)^{n / 2} q(0)=\operatorname{det}\left(\pi_{U}\right) \cdot K^{* 2} .
$$

(c) If $\pi-\operatorname{type}(V)=1^{-}, 1^{+}$or $2^{-}$then $\pi \in \Omega(V)$.

(d) If $\pi-\operatorname{type}(V)=2^{+}$then $\Theta(\pi)=\mathrm{d}(V)$.

Proof. Let $\pi-\operatorname{type}(V) \in\left\{2^{*}, 3\right\}$. Then $\operatorname{dim}(V)$ is even and $\mathrm{B}^{\infty}(\pi)=V=$ $\mathrm{B}^{\infty}(-\pi)$. From Lemma 4.3 we obtain $\mathrm{d} V=\Theta(-1)=\Theta(\pi) \Theta(-\pi)=\operatorname{det}(1-$ $\pi) \cdot \operatorname{det}(1+\pi) \cdot K^{* 2}=p(1) p(-1) K^{* 2}$. Hence we obtain (as $\operatorname{dim}(V)$ is even) $\Theta(\pi)=\operatorname{det}((1-\pi) / 2) \cdot \mathrm{d} V=\operatorname{det}(1-\pi) \cdot \mathrm{d} V=p(-1) K^{* 2}$. Now consider type 3 only. Let $m:=n / 2$ and $q=x^{m}+\cdots+a_{0}$. Then $\Theta(\pi)=p(-1) K^{* 2}=q(-1) q^{*}(-1) K^{* 2}=$ $\left((-1)^{m}+a_{m-1}(-1)^{m-1}+\cdots+a_{0}\right)^{2}(-1)^{m} a_{0} \cdot K^{* 2}=(-1)^{m} a_{0} \cdot K^{* 2}=\operatorname{det}\left(\pi_{U}\right) \cdot K^{* 2}$.

If $\pi$-type $(V)=1^{-}$then $\pi$ is unipotent; hence $\pi \in \operatorname{ker}(\Theta)$ by Lemma 4.3. If $\pi-\operatorname{type}(V)=1^{+}$then $(-\pi)-\operatorname{type}(V)=1^{-}$, hence $\Theta(-\pi)=K^{* 2}$ by the previous argument. Furthermore, $V$ is the orthogonal sum of an even number of hyperbolic planes (cf. Definition 3.5), hence $\Theta\left(-1_{V}\right)=K^{* 2}$. We obtain $\Theta(\pi)=K^{* 2}$. In both cases $\operatorname{dim}(\mathrm{B}(\pi))$ is even. Hence $\pi \in \Omega(V)$. Also if $\pi-\operatorname{type}(V)=2^{-}$then $\pi$ is unipotent and $\operatorname{dim}(B(\pi))$ is even.

LEMMA 4.8. Let $\pi \in \mathrm{O}(V)$ and $\pi-\operatorname{type}(V)=2^{*}$. Suppose that $\operatorname{mip}(\pi)$ is not a square (in $K[x]$ ), and $K$ is finite or $K=\mathbb{R}$. Then $V$ is not a hyperbolic space.

PROOF. See $[5,4.1]$.

LEMMA 4.9. Let $n=2, \pi \in \mathrm{O}(V)$ and $\pi-\operatorname{type}(V)=2^{*}$. Then $V$ is anisotropic.

PROOF. We have $q:=\operatorname{mip}(\pi)=x^{2}+\alpha x+1$ for some $\alpha \in K$. As $\operatorname{mip}(\pi)$ is irreducible it follows that $\alpha^{2}-4 \notin K^{* 2}$. The characteristic polynomials of $1-\pi$, respectively $1+\pi$, are $q(1-x) \in K[x]$, respectively $q(x-1)$. As $\mathrm{B}^{\infty}(\pi)=V$ we obtain from Lemma 4.3 that $\Theta(\pi)=\operatorname{det}(1-\pi) \mathrm{d} V=q(1) \mathrm{d}(V)=(2+\alpha) \mathrm{d} V$, and $\Theta(-\pi)=q(-1) \mathrm{d}(V)=(2-\alpha) \mathrm{d} V$. Hence, $\mathrm{d} V=\Theta\left(-1_{V}\right)=\Theta(-\pi \pi)=$ $\left(4-\alpha^{2}\right) K^{* 2} \neq-K^{* 2}$. This implies that $V$ is not a hyperbolic plane.

LEMMA 4.10. Suppose that $K$ is finite and $\pi, \psi \in \mathrm{O}(V)$ such that $\mathrm{F}\left(\pi^{2}\right)=0=$ $\mathrm{F}\left(\psi^{2}\right)$. Then $\pi$ is a conjugate of $\psi$ in $\mathrm{O}(V)$ if and only if $\pi$ is a conjugate of $\psi$ in $G L(V)$. 
PROOF. This follows from [20, p. 38].

\section{LEMMA 4.11. Let $n \geq 2$.}

(a) If $U$ is a 1-dimensional subspace of $V$ then $V$ contains at least $(|K|-1) / 2$ [if $V$ is anisotropic then $(|K|+1) / 2$ ] 1-dimensional subspaces $W$ such that $\mathrm{d} W=\mathrm{d} U$. (b) If $\mathrm{u}(K) \leq 2$, then for each $\lambda \in K^{*}, V$ contains at least $(|K|-1) / 2$ [if $V$ is anisotropic then $(|K|+1) / 2$ ] 1-dimensional subspaces $W$ such that $\mathrm{d} W=\lambda K^{* 2}$.

PROOF. See, for example, $[9,3.7]$.

PROPOSITION 4.12 (Scherk's Theorem). [16] Let $\pi \in \mathrm{O}(V)$. Then $\pi$ is a product of $\operatorname{dim}(\mathrm{B}(\pi))$, but not less, symmetries, except when $\mathrm{B}(\pi)$ is totally isotropic. If $\mathrm{B}(\pi)$ is totally isotropic then $\operatorname{dim}(\mathrm{B}(\pi))+2$ symmetries are sufficient and this is the minimal number needed.

LEMMA 4.13. Let $M$ be a finite subset of the field $K, \alpha \in K^{*}$ and $m \in \mathbb{N}_{\geq 2}$. Then degree $(p)=m, p(0)=\alpha$, each zero of $p$ is simple and $M$ does not contain a zero of $p$ for some monic $p \in K[x]$.

PROOF. If $K$ is infinite then one has $\alpha_{1}, \ldots, \alpha_{m} \in K$ such that $p=\left(x-\alpha_{1}\right) \cdots(x-$ $\left.\alpha_{m}\right)$ fulfils the required properties. Let $K$ be finite. Then $|K|^{m-1}$ monic polynomials of degree $m$ satisfy $p(0)=\alpha$. Each $\lambda \in K^{*}$ admits precisely $|K|^{m-2}$ monic polynomials $p$ of degree $m-1$ and such that $p(0)=\alpha / \lambda$; this is also the number of polynomials $q$ of degree $m$ such that $q(\lambda)=0$ and $q(0)=\alpha$. Hence there are at most $(|K|-1)|K|^{m-2}<$ $|K|^{m-1}$ monic polynomials $p$ of degree $m$ such that $p(0)=\alpha$ and $p$ has a zero in $K$. We conclude that at least one monic polynomial of degree $m$ satisfies $p(0)=\alpha$ and has no zero.

\section{Products of orthogonal involutions}

Each orthogonal mapping $\pi \in O(V)$ is a product of two orthogonal involutions. How can we choose the involutions when $\pi$ is given and $V$ is an orthogonally indecomposable $\pi$-module? The following answers are essential tools for our study of $\Omega(V)$.

LEMMA 5.1. Let $\pi=\rho \sigma$ where $\rho$ and $\sigma$ are orthogonal involutions and $\pi-\operatorname{type}(V)$ $=2^{-}$or $\pi-\operatorname{type}(V)=2^{+}$. Let

$$
k:= \begin{cases}\frac{1}{2}(n+1) & \text { if } n \equiv 3 \bmod 4 \\ \frac{1}{2}(n-1) & \text { if } n \equiv 1 \bmod 4\end{cases}
$$


Then one of the following cases occurs:

(a) $\rho \in O^{+}(V)$ and $\mathrm{B}(\rho)$ is a $k$-dimensional hyperbolic space (in particular $\Theta(\rho)=$ $\left.(-1)^{k / 2} K^{* 2}\right)$, or

(b) $\rho \in O^{-}(V)$ and ind $(\mathrm{B}(\rho))=(n-k-1) / 2$ and $\Theta(\rho)=(-1)^{k / 2} \mathrm{~d} V$.

PROOF. If $\pi-\operatorname{type}(V)=2^{+}$and $\pi=\rho \sigma$ then $(-\pi)-\operatorname{type}(V)=2^{-}$and $-\pi=$ $\rho(-\sigma)$. Therefore, it suffices to study the case $\pi-\operatorname{type}(V)=2^{-}$. So let $\pi-\operatorname{type}(V)=$ $2^{-}$. In 4.7 (c) we proved that $\pi \in \Omega(V)$. From Lemma 2.10 it follows that $W:=$ $\operatorname{ker}\left((\pi-1)^{(n-1) / 2}\right)$ is totally isotropic and $\operatorname{dim}(W)=(n-1) / 2$. Hence ind $(V)=$ $(n-1) / 2$ (observe that $n$ is odd; cf. Definition 3.5). We have $W=W \rho=W \sigma=W \pi$ [as $\left((\pi-1)^{(n-1) / 2}\right)^{\rho}=\pi^{-(n-1) / 2}(1-\pi)^{(n-1) / 2}$ ]. Now Lemma 2.7 implies that

(1) $\operatorname{dim}(\mathrm{B}(\rho))=\operatorname{dim}(\mathrm{B}(\sigma)) \in\{(n-1) / 2,(n+1) / 2\}$, and $\left(2^{\prime}\right)$ or $\left(2^{\prime \prime}\right)$ :

(2') If $\operatorname{dim}(W)$ is even, that is, $n \equiv 1 \bmod 4$, then $\operatorname{dim}\left(\mathrm{B}\left(\rho_{W}\right)\right)=\operatorname{dim}\left(\mathrm{B}\left(\sigma_{W}\right)\right)=$ $(n-1) / 4$,

$\left(2^{\prime \prime}\right)$ If $\operatorname{dim}(W)$ is odd, that is, $n \equiv 3 \bmod 4$, then $\operatorname{dim}\left(\mathrm{B}\left(\rho_{W}\right)\right)=\operatorname{dim}\left(\mathrm{B}\left(\sigma_{W}\right)\right) \epsilon$ $\{(n+1) / 4,(n-3) / 4\}$.

Clearly, (1), (2') and (2") imply

(3) $\operatorname{dim}\left(\mathrm{B}\left(\rho_{w}\right)\right)=\operatorname{dim}(\mathrm{B}(\rho)) / 2$ or $\operatorname{dim}\left(\mathrm{F}\left(\rho_{w}\right)\right)=\operatorname{dim}(\mathrm{F}(\rho)) / 2$.

In particular, it follows that

(4) $\mathrm{B}(\rho)$ is a hyperbolic space of dimension $k$ (hence $\left.\Theta(\rho)=(-1)^{k / 2} K^{* 2}\right)$, or $\mathrm{F}(\rho)$ is a hyperbolic space of dimension $k$ and $\operatorname{ind}(\mathrm{B}(\rho))=(\operatorname{dim}(\mathrm{B}(\rho))-1) / 2$ (hence $\left.\Theta(-\rho) \Theta(-1)=\Theta(-\rho) \mathrm{d} V=(-1)^{k / 2} \mathrm{~d} V\right)$.

REMARK 5.2. We refer to the previous lemma.

If case (a) is present, we can replace $\rho, \sigma$ by $-\rho,-\sigma$ and arrive at case (b). The analogue applies when case (b) holds true.

LEMMA 5.3 (type $2^{*}$ and 3 ). Let $\pi=\rho \sigma$ where $\rho$ and $\sigma$ are orthogonal involutions and $\pi-\operatorname{type}(V)=\left(2^{*}\right)$ or $\pi-\operatorname{type}(V)=(3)$. Let $\operatorname{mip}(\pi)=p^{m}$ where $p \in K[x]$. Then $\operatorname{dim}(\mathrm{B}(\rho))=n / 2=\operatorname{dim}(\mathrm{B}(\sigma))$. If $m$ is even then $\mathrm{B}(\rho)$ and $\mathrm{B}(\sigma)$ are hyperbolic spaces. If $m$ is odd then ind $(\mathrm{B}(\rho))$, ind $(\mathrm{B}(\sigma)) \geq(n-\operatorname{degree}(p)) / 4$.

PROOF. The vector space $V$ is a $\pi$-cyclic module. If $m$ is odd, $m=2 t-1$, let $W:=\operatorname{ker}\left(p^{t-1}(\pi)\right)$. If $m$ is even, $m=2 t$, let $W:=\operatorname{ker}\left(p^{t}(\pi)\right)$. Then $W$ is totally isotropic (cf. Lemma 2.10) and invariant under $\pi, \rho$ and $\sigma$ [indeed: $\rho^{-1} p^{t}(\pi) \rho=$ $\left.p^{t}\left(\pi^{\rho}\right)=p^{t}\left(\pi^{-1}\right)=\pi^{t \cdot \text { degree }(p)} p^{t}(\pi)\right]$. We have $\mathrm{N}(\pi)=0=\mathrm{F}(\pi)$ and thus $\operatorname{dim}\left(\mathbf{B}\left(\rho_{W}\right)\right)=\operatorname{dim}(W) / 2=\operatorname{dim}\left(\mathbf{B}\left(\sigma_{W}\right)\right) ; \mathrm{cf}$. Lemma $2.7(\mathrm{a})$. We obtain ind $(\mathbf{B}(\rho)) \geq$ $\operatorname{dim}\left(\mathbf{B}\left(\rho_{W}\right)\right)=\operatorname{dim}(W) / 2$. Clearly, $\sigma$ satisfies the analogous statement. If $m$ is odd we have $\operatorname{dim}(W)=(t-1) \cdot \operatorname{degree}(p)=(n-\operatorname{degree}(p)) / 2$. If $m$ is even then 
$\operatorname{dim}(W)=n / 2$. Furthermore, $\operatorname{dim}(\mathrm{B}(\rho))=n / 2$ by Lemma $2.7(\mathrm{a})$. This yields the assertion.

REMARK 5.4 (real case). Assume that $K=\mathbb{R}, \pi=\rho \sigma$ where $\rho, \sigma$ are orthogonal involutions and $\pi-\operatorname{type}\left(V^{\prime}\right)=2^{*}$. Then $\pi \in \Omega(V)$, and:

If $n \equiv 0 \bmod 4$ then $\operatorname{dim}(\mathrm{B}(\rho))=n / 2=\operatorname{dim}(\mathrm{B}(\sigma))$ and $\mathrm{B}(\rho), \mathrm{B}(\sigma)$ are hyperbolic spaces.

If $n \equiv 2 \bmod 4$ then $\operatorname{dim}(\mathrm{B}(\rho))=n / 2=\operatorname{dim}(\mathrm{B}(\sigma))$ and ind $\mathrm{B}(\rho)=(n-2) / 4=$ ind $\mathrm{B}(\sigma)$ (that is. the maxumum possible value when the dimension is $n / 2$ ).

In each case $\mathbf{B}(\rho)$ W ivimetnc to $\mathrm{B}(\sigma)$.

PROOF. We have $q=$ mipi.T $1=p^{m}$ for an irreducible and symmetric polynomial $p \neq x-1, x+1$. Thus $r=x^{2}+\alpha x+1$ where $\alpha \in \mathbb{R}$ and $q(-1)=p(-1)^{m}$ is positive since all value of $r$ are positive. Now Lemma 4.3 yields that $\Theta(\pi)=1 \cdot K^{* 2}$. Furthermore, $\pi \in \mathbf{O}^{\circ}, 1$, Hence $\pi \in \Omega(V)$. If $m$ is even then the assertion follows immediately from the merinu lemma. Suppose that $m$ is odd. The previous lemma shows that $m / 2=n+\cdots|m| B|\rho| \geq(n-2) / 4$, hence ind $\mathrm{B}(\rho)=(n-2) / 4$. Finally, $\mathrm{dB}(\rho)=\Theta(\rho)=H \mid \cdots)=J H_{1} n$ । implies that $\mathrm{B}(\rho)$ is isometric to $\mathrm{B}(\sigma)$.

LEMMA 5.5 IIyp $\mid$ and $\mid$ Let $\pi=\rho \sigma$ where $\rho$ and $\sigma$ are orthogonal invo-

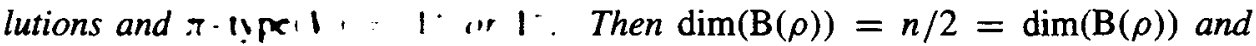
$\mathrm{B}(\rho), \mathrm{B}(\sigma)$ are hipreruils. in. $\mathrm{Cl}$ In particular, $\rho, \sigma \in \Omega(V)$.

PROOF. Let $7 \cdot 1 \frac{1}{1} \quad !$ hence $\operatorname{mip}(\pi)=p^{k}$ where $p=x-1, k$ is even and $n=2 k$.

The same argument a in the proof of Lemma 5.3 yield that $Z:=\operatorname{ker}(\pi-1)^{k / 2}$ is totally isotropic and inveriant under $\rho$ and $\sigma$. Clearly, $\operatorname{dim}(Z)=k$. From Lemma 2.7 we obtain $\operatorname{dim}(\mathbf{B}(\rho))=,\operatorname{dim}\left(\mathbf{B}\left(\sigma_{z}\right)\right) \leq \operatorname{dim}(\mathbf{B}(\rho)) / 2$ as $\mathbf{N}(\pi)=0$ and $\mathbf{B}(\rho)$ is regular]. Furthermore. $\operatorname{dim}\left(F\left(\rho_{2}\right)\right) \leq \operatorname{dim}(F(\rho)) / 2$ as $\mathrm{F}(\rho)$ is regular. So we obtained $k=\operatorname{dim}(Z)=\operatorname{dim}\left(\mathrm{B}\left(\rho_{l}\right)\right)+\operatorname{dim}\left(\mathrm{F}\left(\rho_{Z}\right)\right) \leq \operatorname{dim}(\mathrm{B}(\rho)) / 2+\operatorname{dim}(\mathrm{F}(\rho)) / 2=$ $n / 2=k$. Therefore. $\operatorname{dim}\left(\mathrm{B}\left(\rho_{Z}\right)\right)=\operatorname{dim}(\mathrm{B}(\rho)) / 2$ and $\mathrm{B}(\rho)$ is a hyperbolic space. Similarly, $\mathbf{F}(\rho)), \mathbf{B}(\sigma))$ and $\mathbf{F}(\sigma)$ are hyperbolic spaces. We have $m:=\operatorname{dim}(\mathbf{B}(\rho))=$ $\operatorname{dim}(\mathrm{B}(\sigma))$; cf. Lemma 2.7. As $2(k-1)=n-2=\operatorname{dim}(\mathrm{B}(\pi)) \leq 2 m$ (cf. Lemma 2.6) and $k, m$ are both even numbers we obtain $k \leq m$. Furthermore, $\operatorname{dim}(\mathrm{F}(\pi))=2$ implies that $m \leq k+1$; cf. Lemma 2.7. We conclude that $m=k$.

Analogous arguments apply when $\pi$-type $(V)=1^{+}$.

LEMMA 5.6. Suppose that $\pi, \varphi \in \mathrm{O}(V)$ are mappings such that $\pi$-type $(V)=3=$ $\varphi$-type $(V)$ and $\operatorname{mip}(\pi)=\operatorname{mip}(\varphi)$. Then $\pi^{\alpha}=\varphi$ for some $\alpha \in \mathrm{O}(V)$. 
PROOF. Let $p=q q^{*}$ be the minimum polynomial of $\pi$ and $\varphi$. Choose $u, z \in V$ such that $\operatorname{ker}(q(\pi))=\langle u\rangle_{\pi}$ and $\operatorname{ker}(q(\varphi))=\langle z\rangle_{\varphi}$. We have $V=\operatorname{ker}(q(\pi)) \oplus \operatorname{ker}\left(q^{*}(\pi)\right)=$ $\operatorname{ker}(q(\varphi)) \oplus \operatorname{ker}\left(q^{*}(\varphi)\right)$ and each direct summand is totally isotropic and has dimension $n / 2$. Define a linear mapping $\alpha: \operatorname{ker}(q(\pi)) \rightarrow \operatorname{ker}(q(\varphi)), u \pi^{j} \mapsto w \pi^{j}$. Then $\alpha$ is a bijection and $\left.\pi\right|_{\operatorname{ker}(q(\pi))}=\alpha\left(\left.\varphi\right|_{\operatorname{ker}(q(\varphi))}\right) \alpha^{-1}$. Obviously, there is an extension $\bar{\alpha} \in \mathrm{O}(V)$ of $\alpha$ that maps $\operatorname{ker}\left(q^{*}(\pi)\right)$ onto $\operatorname{ker}\left(q^{*}(\varphi)\right)$. Hence $\pi$ and $\tilde{\alpha} \varphi \tilde{\alpha}^{-1}$ are both orthogonal mappings of $V$ which leave $\operatorname{ker}\left(q^{*}(\pi)\right)$ invariant and whose restrictions to $\operatorname{ker}(q(\pi))$ coincide. Hence they are equal; cf. Lemma 4.4.

LEMMA 5.7 (type 3). Let $\pi \in \mathrm{O}(V), \pi-\operatorname{type}(V)=3$ and $\operatorname{mip}(\pi)=q q^{*}$. Let $\lambda \in$ $K^{*}$. Then $\pi=\rho \sigma$ for involutions $\rho, \sigma \in \mathrm{O}(V)$ with $\operatorname{dim}(\mathrm{B}(\rho))=n / 2=\operatorname{dim}(\mathrm{B}(\sigma))$ and the following additional properties.

(i) If $n \equiv 0 \bmod 4$ then $\mathrm{B}(\rho)$ is a hyperbolic space and $\Theta(\rho)=(-1)^{n / 4} K^{* 2}$ and ind $\mathrm{B}(\sigma) \geq n / 4-1$.

(ii) If $n \equiv 2 \bmod 4$ then ind $\mathrm{B}(\rho)=$ ind $\mathrm{B}(\sigma)=(n-2) / 4$ (hence the maximum possible for a regular space of dimension $n / 2)$; furthermore, $\Theta(\rho)=\lambda K^{* 2}$.

PROOF. The statement $\operatorname{dim}(\mathrm{B}(\rho))=n / 2=\operatorname{dim}(\mathrm{B}(\sigma))$ follows from Lemma 2.7. Our type-classification yields $V=U \oplus W$ where $U:=\operatorname{ker}(q(\pi))$ and $W:=$ $\operatorname{ker}\left(q^{*}(\pi)\right) ; U$ and $W$ are totally isotropic. Take a basis $u_{1}, \ldots, u_{m}$ for $U$ and a basis $w_{1}, \ldots, w_{m}$ for $W$ such that $f\left(u_{i}, w_{m-j}\right)=\delta(i, j) \cdot \mu$ where $\mu:=2 \lambda(-1)^{m(m+1) / 2}$. Define $\psi \in \mathrm{GL}(U)$ such that $u_{i} \mapsto u_{i+1}$ for $i=1, \ldots, m-1$ and $u_{m} \mapsto$ $-a_{m-1} u_{m}-\cdots-a_{0} u_{1}$ where $q=x^{m}+a_{m-1} x^{m-1}+\cdots+a_{0}$. Then $\operatorname{mip}(\psi)=q$. According to Lemma $4.4 \psi$ admits a unique extension to a mapping in $O(V)$ which leaves $W$ invariant. Call this mapping $\psi$ also. Then $\operatorname{mip}(\psi)=q q^{*}$ [as $\operatorname{mip}(\psi)$ is symmetric and $q$ is prime to $q^{*}$ ] and $\psi$ is a conjugate of $\pi$ in $\mathrm{O}(V)$ by the previous lemma. Hence we can a priori assume that $\pi=\psi$. Let $u:=u_{1}$ and $w:=w_{1}$. Define $\rho \in \mathrm{GL}(V)$ by the properties $u \psi^{i} \mapsto w \psi^{-i}$ and $w \psi^{-i} \mapsto u \psi^{i}$ for $i=0, \ldots, m-1$. Then $\rho \in \mathrm{O}(V)$; furthermore, $\rho$ and $\sigma:=\rho \psi$ are involutions and $\mathrm{B}(\rho)=\left\langle w \psi^{-i}-u \psi^{i} \mid i \in\{0, \ldots, m-1\}\right\rangle$. We obtain $f\left(w \psi^{-i}-u \psi^{i}, w \psi^{-j}-u \psi^{j}\right)=-2 f\left(u \psi^{i}, w \psi^{-j}\right)=-2 f\left(u \psi^{i+j}, w\right)$. This equals 0 if $0 \leq i+j<m-1$ and $-2 \mu$ if $i+j=m-1$. Hence ind $\mathrm{B}(\rho)$ attains the maximal possible value. If $m$ is even then $\mathrm{B}(\rho)$ is a hyperbolic space; hence $\Theta(\rho)=\mathrm{dB}(\rho)=$ $(-1)^{m / 2} K^{* 2}$. If $m$ is odd we obtain that $\Theta(\rho)=\mathrm{dB}(\rho)=\lambda K^{* 2}$. Furthermore, $\mathrm{B}(\sigma)=\left\langle w \psi^{1-i}-u \psi^{i} \mid i \in\{0, \ldots, m-1\}\right\rangle$ and for $i, j \in\{0, \ldots, m-1\}$ one has $f\left(w \psi^{1-i}-u \psi^{i}, w \psi^{1-j}-u \psi^{j}\right)=-2 f\left(w, u \psi^{i+j-1}\right)$. This is 0 if $0 \neq i+j<m$. Hence ind $\mathrm{B}(\sigma)$ attains the maximal possible value if $m$ is odd. If $m$ is even we obtain ind $\mathrm{B}(\sigma) \geq m / 2-1$. 
LemMa 5.8. Let $n \equiv 2 \bmod 4$ and $\pi \in \mathrm{O}(V)$ and $\lambda \in K^{*}$. Suppose that $\pi-\operatorname{type}(V)=3$ or $\left[K\right.$ finite and $\left.\pi-\operatorname{type}(V)=2^{*}\right]$. Then $\pi=\rho \sigma$ and $\Theta(\rho)=\lambda K^{* 2}$ for some involutions $\rho, \sigma \in \mathrm{O}(V)$.

ProOF. Take involutions $\omega, \eta \in \mathrm{O}(V)$ such that $\pi=\omega \eta$. Choose $\mu \in \lambda \Theta(\omega)$. As $\mathrm{u}(K) \leq 2$ or $V$ is a hyperbolic space we can obviously find $\varphi \in \mathrm{GL}(V)$ such that $f(v \varphi, w \varphi)=\mu f(v, w)$ for all $v, w \in V$. Then $\pi^{\varphi}, \omega^{\varphi}, \eta^{\varphi} \in \mathrm{O}(V)$. If $\pi$-type $=$ 3 then Lemma 5.6 supplies $\alpha \in \mathrm{O}(V)$ such that $\pi=\pi^{\varphi \alpha}$. If $K$ is finite and $\pi-\operatorname{type}(V)=2^{*}$ then Lemma 4.10 yields the same result. Let $\rho:=\omega^{\varphi \alpha}$ and $\sigma:=\eta^{\varphi \alpha}$. Then $\rho, \sigma \in \mathrm{O}(V)$, both elements are involutions, $\pi=\rho \sigma$ and $\Theta(\rho)=\mathrm{dB}(\rho)=$ $\mathrm{d}(\mathrm{B}(\omega) \varphi \alpha)=\mathrm{d}(\mathrm{B}(\omega) \varphi)=\mu^{n / 2} \mathrm{~dB}(\omega)=\mu \Theta(\omega)=\lambda K^{* 2}$ as $n \equiv 2 \bmod 4$.

COROLlARY 5.9. Let $\pi=\rho \sigma$ where $\rho, \sigma \in \mathrm{O}(V)$ are involutions and $V$ is an orthogonally indecomposable $\pi$-module.

(a) If $\pi-\operatorname{type}(V) \notin\left\{2^{+}, 2^{-}\right\}$then $\operatorname{dim}(\mathrm{B}(\rho))=n / 2=\operatorname{dim}(\mathrm{B}(\sigma))$.

(b) If $\pi-\operatorname{type}(V)=2^{-}$then $\operatorname{dim}(\mathrm{B}(\rho))=(n-1) / 2=\operatorname{dim}(\mathrm{B}(\sigma))$ or $\operatorname{dim}(\mathrm{B}(\rho))=$ $(n+1) / 2=\operatorname{dim}(\mathrm{B}(\sigma))$, and we can achieve each of the two possibilities.

(c) If $\pi-\operatorname{type}(V)=2^{+}$then $\operatorname{dim}(\mathrm{B}(\rho))=(n-1) / 2$ and $\operatorname{dim}(\mathrm{B}(\sigma))=(n+1) / 2$, or $\operatorname{dim}(\mathrm{B}(\rho))=(n+1) / 2$ and $\operatorname{dim}(\mathrm{B}(\sigma))=(n-1) / 2$; we can achieve each of the two possibilities.

Unless $\pi-\operatorname{type}(V)=2^{+}$it follows that $\operatorname{dim}(\mathrm{B}(\rho))=\operatorname{dim}(\mathrm{B}(\sigma))$. Furthermore, $\operatorname{det}(\pi)=-1$ if and only if $\pi-\operatorname{type}(V)=2^{+}$.

This follows immediately from Lemmas $2.7,5.1$ and 5.5.

COROLlaRY 5.10. Let $\pi \in \mathrm{O}(V)$ and $s \in \mathbb{N} \cap\{(n-1) / 2, n / 2,(n+1) / 2\}$. One has $\pi=\rho \sigma$ for involutions $\rho, \sigma \in \mathrm{O}(V)$.

(a) If $\pi \in \mathrm{O}^{+}(V)$ one can achieve that $\operatorname{dim}(\mathrm{B}(\rho))=s=\operatorname{dim}(\mathrm{B}(\sigma))$.

(b) If $\pi \in \mathrm{O}^{-}(V)$ one can achieve that $\operatorname{dim}(\mathrm{B}(\rho))=\lceil n / 2\rceil+1$ and $\operatorname{dim}(\mathrm{B}(\sigma))=$ $\lceil n / 2\rceil$.

A decomposition of $V$ into orthogonally indecomposable $\pi$-modules contains at least $|\operatorname{dim}(\mathrm{B}(\rho))-\operatorname{dim}(\mathrm{B}(\sigma))| \pi$-modules of type $2^{+}$.

PROOF. If $\pi \in \mathrm{O}^{+}(V)$ then an orthogonal decomposition of $V$ into orthogonally indecomposable $\pi$-modules contains an even number of modules of $\pi$-type $=2^{+}$; if $\pi \in \mathrm{O}^{-}(V)$ then this number is odd. Hence the assertion follows from the previous corollary. 


\section{Symmetries in the kernel of the spinorial norm}

Let $S$ denote the set of symmetries in $\operatorname{ker}(\Theta)$. We want to write a given $\pi \in \operatorname{ker}(\Theta)$ as a product of as few as possible symmetries in $\mathrm{S}$. This problem was studied within a more general framework in [9]. We quote a result.

Proposition 6.1 (cf. [9], Corollaries I2 and I3). Let $|K|>7, V$ be isotropic, $\pi \in$ $\operatorname{her}(\Theta)$ and $k:=\operatorname{dim}(\mathrm{B}(\pi))$.

(a) If $\pi^{2} \neq 1$ and $\mathrm{B}(\pi) / \operatorname{rad}(\mathrm{B}(\pi)$ ) is isotropic (that is, there is an isotropic vector $=0$ in $\mathrm{B}(\pi) \backslash \operatorname{rad}(\mathrm{B}(\pi)))$ then $\pi$ is a product of $k$ but not less than $k$ symmetries in $S$. (b) $\pi$ is a product of $k+2$ elements of $S$

From the previous proposition one obtains:

COROLlaRY 6.2. Suppose that $u(K) \leq 2$ and $|K| \geq 5$. Then $S$ generates $\operatorname{ker}(\Theta)$.

(a) Let $\pi \in \operatorname{ker}(\Theta), k:=\operatorname{dim}(\mathrm{B}(\pi))$ and $\operatorname{dim}(\mathrm{B}(\pi) / \operatorname{rad}(\mathrm{B}(\pi)) \geq 2$. Then $\pi$ is a prinducl of $k$ but not less than $k$ symmetries of $S$.

(h) Let $\pi \in \operatorname{ker}(\Theta), k:=\operatorname{dim}(\mathrm{B}(\pi))$ and $\operatorname{dim}(\mathrm{B}(\pi) / \operatorname{rad}(\mathrm{B}(\pi))=1$. If $\mathrm{d}(\mathrm{B}(\pi) /$ rad $(\mathrm{B}(\mathrm{T}))=K^{* 2}$ then $\pi$ is a product of $k$ but not less than $k$ symmetries of $S$; "thenise one needs $k+2$ symmetries in $S$.

(i) Let $\pi \in \operatorname{ker}(\Theta), k:=\operatorname{dim}(\mathrm{B}(\pi))$ and $\mathrm{B}(\pi)$ be totally isotropic. Then $\pi$ is a priducl of $k+2$ but not less than $k+2$ symmetries of $S$.

R! VARK 6.3. Let $|K|=3$.

(d) If $n \in\{3,4\}$ and $d V=K^{* 2}$ then $S$ does not generate $\operatorname{ker}(\Theta)$.

1h. If $n \geq 5$ or if $\mathrm{d} V \neq K^{* 2}$ then $\mathrm{S}$ generates $\operatorname{ker}(\Theta)$; however, it is not so easy to und the minimal number of factors needed. For example, if $n \geq 5$ one can find some $7: \operatorname{her}(\Theta)$ where $\operatorname{dim}(\mathrm{B}(\pi))+4$ symmetries in $\operatorname{ker}(\Theta)$ are needed.

\section{Reflections in $\Omega(V)$ and $\operatorname{ker}(\Theta)$}

LeMMA 7.1. Let $|K| \geq 4$. Then $P S L_{2}(K)$ is 3-reflectional. $P S L_{2}(K)$ is 2reflectional if and only if $\mathrm{s}(K)=1$.

PROOF. Let denote the canonical homomorphism of $\mathrm{SL}_{2}(K)$ onto $\mathrm{PSL}_{2}(K)$. For $\lambda, \mu \in K$ where $\lambda \neq 0$ let

$$
\rho(\mu, \lambda):=\left(\begin{array}{cc}
\mu & \lambda \\
-\lambda^{-1}\left(1+\mu^{2}\right) & -\mu
\end{array}\right)
$$


Then $\rho(\mu, \lambda) \in \mathrm{SL}_{2}(K)$ and $\tilde{\rho}$ is an involution. Furthermore, if $\mathbf{s}(K) \neq 1$ then each involution $\neq \tilde{1}$ in $\operatorname{PSL}_{2}(K)$ has this form. Now let $\pi \in \mathrm{SL}_{2}(K)$ be such that $\tilde{\pi} \neq \tilde{1}$. Then (up to similarity)

$$
\pi=\left(\begin{array}{cc}
0 & 1 \\
-1 & \kappa
\end{array}\right)
$$

for some $\kappa \in K$. If $\kappa=0$ then $\tilde{\pi}$ is an involution. So let $\kappa \neq 0$. If $\mathrm{s}(K) \neq 1$ take $\lambda^{2} \neq 1$. Then

$$
\pi=\rho\left(-\lambda,(\lambda \kappa)^{-1}\left(\lambda^{2}-1\right)\right) \cdot \rho\left(1,\left(\lambda^{2} \kappa\right)^{-1}\left(1-\lambda^{2}\right)\right) \cdot \rho\left(0, \lambda^{-1}\right) .
$$

If $\mathrm{s}(K)=1$ then take $\lambda \in K$ such that $\lambda^{2}=-1$. Then $\pi=\rho(0, \lambda) \rho(\lambda, \lambda \kappa)$.

If $s(K) \neq 1$ check that

$$
\tau=\left(\begin{array}{cc}
0 & 1 \\
-1 & 2
\end{array}\right)
$$

is not a product of two $\rho$ 's; hence $\tilde{\tau}$ is not a product of two involutions in $\operatorname{PSL}_{2}(K)$.

COROLLARY 7.2. (a) If $n=3, V$ is isotropic and $|K| \neq 3$, then $\Omega(V)$ is 3reflectional; $\Omega(V)$ is 2-reflectional if and only if $\mathrm{s}(K)=1$.

(b) Let $n=4$ and $\operatorname{ind}(V)=1$. Then $\Omega(V)$ is 3-reflectional; $\Omega(V)$ is 2-reflectional if and only if $\mathrm{s}(K)=1$ or $\mathrm{d} V=-K^{* 2}$.

PROOF. (a) We know that $\Omega(V) \cong \mathrm{PSL}_{2}(K)$; cf. Lemma 4.2. Hence the previous lemma yields the assertion.

(b) As $\Omega(V) \equiv \mathrm{PSL}_{2}\left(K(\delta)\right.$ ) where $\delta^{2} K^{* 2}=\mathrm{d} V$ (cf. Lemma 4.2) the previous lemma yields the assertion.

LEMMA 7.3. Let $\mathrm{u}(K)<\infty$ and $\lambda \in K^{*}$. Let $\omega \in \mathrm{O}(V)$ be an involution such that $\operatorname{dim}(\mathrm{B}(\omega)) \geq \mathbf{u}(K)$ or $\operatorname{dim}(\mathrm{F}(\omega)) \geq \mathbf{u}(K)$. Then:

(a) Some symmetry $\rho \in \mathrm{O}(V)$ satisfies $\Theta(\rho)=\lambda K^{* 2}$ and $\rho \omega$ is an involution.

(b) If $\omega \in\{1,-1\}$ suppose additionally that $n \geq \mathrm{u}(K)+1$. Some involution $\rho \in \mathrm{O}(V)$ then satisfies $\operatorname{dim}(\mathrm{B}(\rho))=2$ and $\Theta(\rho)=\lambda K^{* 2}$ and $\rho \omega$ is an involution.

PROOF. We may assume that $\operatorname{dim}(\mathrm{B}(\omega)) \geq \mathrm{u}(K)$ (otherwise replace $\omega$ by $-\omega$ ). (a) Choose $v \in \mathrm{B}(\omega)$ such that $q(v)=\lambda$. Then the symmetry $\rho$ whose path is $\langle v\rangle$ fulfils the requirements. (b) If $\omega=-1$, take any anisotropic vector $v \in V$. As $\operatorname{dim}\left(v^{\perp}\right) \geq \mathbf{u}(K)$ we find $w \in v^{\perp}$ such that $q(w)=\lambda q(v)$. The orthogonal involution $\rho$ whose path is $\langle v, w\rangle$ meets the requirements. Now let $\omega \neq-1$. Take an anisotropic vector $z \in \mathrm{F}(\omega)$. As $\operatorname{dim}(\mathrm{B}(\omega)) \geq \mathrm{u}(K)$ we find $y \in \mathrm{B}(\omega)$ such that $q(y)=\lambda q(z)$. Let $\rho \in \mathrm{O}(V)$ be the involution with $\mathrm{B}(\rho)=\langle z, y\rangle$. 
LEMMA 7.4. Suppose $2 \leq \mathrm{u}(K)<\infty$. Let $\pi=\rho \sigma$ where $\rho$ and $\sigma$ are orthogonal involutions.

(a) If $n \geq 2 \mathrm{u}(K)+2$ and $\operatorname{dim}(\mathrm{B}(\rho))=1$, then $\pi=\rho^{\prime} \sigma^{\prime}$, where $\rho^{\prime}$ and $\sigma^{\prime}$ are orthogonal involutions and $\rho^{\prime} \in \Omega(V)$.

(b) If $n \geq 2 \mathrm{u}(K)+4$ and $\operatorname{dim}(\mathrm{B}(\rho))=2$ then $\pi=\rho^{\prime} \sigma^{\prime}$ where $\rho^{\prime}$ and $\sigma^{\prime}$ are orthogonal involutions and $\rho^{\prime} \in \Omega(V)$.

PROOF. As $n \geq 2 \mathrm{u}(K)+1$ we have ind $(V) \geq 1$; hence $\Omega(V)=\operatorname{ker}(\Theta) \cap \mathrm{O}^{+}(V)$. Furthermore, $\pi^{2}=\rho \rho^{\sigma}$.

(a) We observed that $\pi^{2}$ is a product of two symmetries. Hence $\operatorname{dim}\left(\mathrm{B}\left(\pi^{2}\right)\right) \leq 2$ and $\operatorname{dim}\left(\operatorname{rad}\left(\mathrm{B}\left(\pi^{2}\right)\right)\right) \leq 1$. This yields that $\operatorname{dim}\left(\mathrm{F}\left(\pi^{2}\right)\right) \geq n-2$ and $\operatorname{dim}\left(\operatorname{rad}\left(\mathrm{F}\left(\pi^{2}\right)\right)\right) \leq 1$. Furthermore, $\mathrm{F}\left(\pi^{2}\right)=\mathrm{F}(\pi) \oplus \mathrm{N}(\pi)$. Therefore, we find a regular subspace $U$ such that $\operatorname{dim}(U) \geq n-3, U$ is a $\pi$-module and $\pi_{U}$ is an involution. We know that $\pi_{U^{\perp}}=\rho_{1} \sigma_{1}$ for involutions $\rho_{1}, \sigma_{1} \in \mathrm{O}\left(U^{\perp}\right)$ (2-reflectionality of orthogonal groups). Now $\omega:=\pi_{U}$ is an involution and $\operatorname{dim}(\mathrm{B}(\omega)) \geq \mathbf{u}(K)$ or $\operatorname{dim}(\mathrm{F}(\omega)) \geq \mathrm{u}(K)$ (as $\operatorname{dim}(U) \geq 2 \mathrm{u}(K)-1$ ). Hence Lemma 7.3 supplies an involution $\rho_{2}$ such that $\sigma_{2}:=\rho_{2} \omega$ is an involution with spinorial norm equal to $\Theta\left(\rho_{1}\right)$ and additionally that $\operatorname{dim}\left(\mathrm{B}\left(\rho_{2}\right)\right)=1$ if $\operatorname{dim}\left(\mathrm{B}\left(\rho_{1}\right)\right)$ is odd, $\operatorname{dim}\left(\mathrm{B}\left(\rho_{2}\right)\right)=2$ if $\operatorname{dim}\left(\mathrm{B}\left(\rho_{1}\right)\right)$ is even. Thus $\pi_{U}=\rho_{2} \sigma_{2}$. Let $\rho^{\prime}:=\rho_{1}(1) \rho_{2}$ and $\sigma^{\prime}:=\sigma_{1}(1) \sigma_{2}$. Then $\pi=\rho^{\prime} \sigma^{\prime}$ and $\rho^{\prime} \in \operatorname{ker}(\Theta) \cap \mathrm{O}^{+}(V)$.

(b) We have $\operatorname{dim}\left(\mathrm{B}\left(\pi^{2}\right)\right) \leq 4$ and $\operatorname{dim}\left(\operatorname{rad}\left(\mathrm{B}\left(\pi^{2}\right)\right)\right) \leq 2$ (see the previous section). So we obtain a regular subspace $U$ such that $\pi_{U}$ is an involution and $\operatorname{dim}(U) \geq n-6$. Choose a maximal $\pi$-module $U$ with these properties.

Case 1: $\operatorname{dim}(U) \geq n-5 \geq 2 \mathrm{u}(K)-1$. Then the analogue arguments as in the proof of (a) yield the assertion.

Case 2: $\operatorname{dim}(U)=n-6$. Then $\operatorname{dim}\left(\mathrm{B}\left(\pi^{2}\right)\right)=4$ and $\operatorname{dim}\left(\operatorname{rad}\left(\mathrm{B}\left(\pi^{2}\right)\right)=2\right.$. Inspection of the types of orthogonally indecomposable $\pi^{2}$-modules yields the following possibilities for

$V=A \oplus B \oplus C$ : either $(1) \pi^{2}-\operatorname{type}(A)=1^{-}, \operatorname{dim}(A)=4$ and $\mathrm{B}\left(\pi^{2}{ }_{B}\right)=B$ 2-dimensional and $\pi_{C}{ }^{2}=1_{C}$; or

(2) $\pi^{2}-\operatorname{type}(A)=2^{-}$and $\operatorname{dim}(A)=3$, the same holds true for $B$ and $\pi_{C}{ }^{2}=1_{C}$.

As $\pi^{2}$ is a product of two involutions with 2-dimensional paths $\left(\pi^{2}=\rho \rho^{\sigma}\right)$ we conclude that only (2) is possible; cf. Corollary 5.9 and Proposition 4.6. Hence $\operatorname{mip}\left(\pi^{2}\right)=(x-1)^{3}$ and therefore $U^{\perp}$ decomposes into two 3-dimensional $\pi$ modules of type $2^{-}$or $2^{+}$. Using Lemma 5.1 and Remark 5.2 we obtain involutions $\rho_{1}, \sigma_{1} \in \mathrm{O}\left(U^{\perp}\right)$ such that $\pi_{U^{\perp}}=\rho_{1} \sigma_{1}$ and $\mathrm{B}\left(\rho_{1}\right)$ is a 4-dimensional hyperbolic space. Thus, $\rho_{1} \in \Omega\left(U^{\perp}\right)$. The involutions $\rho^{\prime}:=\rho_{1} \oplus 11_{U}$ and $\sigma^{\prime}:=\sigma_{1} \oplus \pi_{U}$ fulfil our requirements. 
THEOREM 7.5. Let $2 \leq \mathrm{u}(K)<\infty$.

(a) Let $n \geq 2 \mathrm{u}(K)+2$. Then $\operatorname{ker}(\Theta)$ is 3-reflectional.

(b) Let $n \geq 2 \mathrm{u}(K)+3$, or $[\mathrm{u}(K)$ even and $n \geq 2 \mathrm{u}(K)+2]$. Then $\Omega(V)$ is 3-reflectional.

PROOF. As $n \geq \mathrm{u}(K), V$ is isotropic. Hence $\Omega(V)=\operatorname{ker}(\Theta) \cap \mathrm{O}^{+}(V)$. Let $\pi \in \operatorname{ker}(\Theta)$. Then Corollary 5.10 provides involutions $\rho, \sigma \in \mathrm{O}(V)$ such that $\pi=\rho \sigma$ and $k:=\operatorname{dim}(B(\rho)) \geq n / 2$; if $\pi \in \mathrm{O}^{+}(V)$ then $k=n / 2$ or $k$ is odd.

Proof of (a). Lemma 7.3 supplies a symmetry $\kappa$ such that $\rho \kappa$ is an involution and $\rho \kappa \in \operatorname{ker}(\Theta)$. The previous lemma provides involutions $\rho^{\prime} \in \operatorname{ker}(\Theta)$ and $\sigma^{\prime} \in \mathrm{O}(V)$ such that $\kappa \sigma=\rho^{\prime} \sigma^{\prime}$. As $\kappa \sigma \in \operatorname{ker}(\Theta)$ it follows that $\sigma^{\prime} \in \operatorname{ker}(\Theta)$. We have $\pi=(\rho \kappa) \rho^{\prime} \sigma^{\prime}$.

Proof of (b). If $k$ is even then our assumptions imply that $n \geq 2 \mathrm{u}(K)+4$. From Lemma 7.3 we get an involution $\kappa \in \mathrm{O}(V)$ such that $\operatorname{dim}(\mathrm{B}(\kappa)) \leq 2$ and $\rho \kappa$ is an involution in $\Omega(V)$. So $\operatorname{dim}(\mathrm{B}(\kappa))=2$ if and only if $k$ is even. Hence $\kappa \sigma \in \Omega(V)$, and the previous lemma supplies involutions $\rho^{\prime}, \sigma^{\prime} \in \Omega(V)$ such that $\kappa \sigma=\rho^{\prime} \sigma^{\prime}$. We obtained $\pi=(\rho \kappa) \rho^{\prime} \sigma^{\prime}$ and proved that $\Omega(V)$ is 3-reflectional.

COROLLARY 7.6. Let $\mathrm{u}(K)=2$ and $n \geq 6$. Then $\Omega(V)$ and also $\operatorname{ker}(\Theta)$ are 3-reflectional.

For finite fields $K$ we will also study the small dimensions $n=2,3,4,5$ which are not subsumed in the previous corollary.

\section{Special cases: finite and euclidean fields}

Let $n \geq 3$. Then $\mathrm{O}^{+}(V)$ is 3-reflectional; $\mathrm{O}^{+}(V)$ is 2-reflectional if and only if $n \not \equiv 2 \bmod 4$; cf. [8].

If $K$ is a euclidean field and $V$ is anisotropic, or if $u(K)=1$, then obviously $\Omega(V)=\mathrm{O}^{+}(V)$. Hence we obtain:

PROPOSITION 8.1. Let $n \geq 3$. Suppose that $K$ is a euclidean field and $V$ is anisotropic, or $\mathrm{u}(K)=1$. Then $\Omega(V)$ is 3-reflectional. Furthermore, $\Omega(V)$ is 2 -reflectional if and only if $n \not \equiv 2 \bmod 4$.

LEMMA 8.2. (a) Suppose that $n=2$ and $[|K| \neq 3$ or $V$ is not a hyperbolic plane $]$. Then $O^{+}(V) \neq\{1,-1\}$.

(b) Suppose that $n$ is odd and $\operatorname{ind}(V)=(n-1) / 2$. Then some $\pi \in \mathrm{O}(V)$ satisfies $\pi-\operatorname{type}(V)=2^{-}\left(\right.$hence $\left.(-\pi)-\operatorname{type}(V)=2^{+}\right)$. 
(c) Let $V$ be a hyperbolic space, $q$ a polynomial of degree $n / 2$ and a power of an irreducible monic polynomial such that $q$ is prime to $q^{*}$. Then some $\pi \in O(V)$ satisfies $\pi-\operatorname{type}(V)=3$ where $\operatorname{mip}(\pi)=q q^{*}$.

(d) Suppose that $V$ is hyperbolic and $n \equiv 0 \bmod 4$. Then some $\pi \in \mathrm{O}(V)$ satisfies $\pi-\operatorname{type}(V)=1$.

PROOF. (a) Take an anisotropic $a \in V$ and an anisotropic $b \in V \backslash\left(\langle a\rangle \cup a^{\perp}\right)$. Then $\pi:=\sigma_{a} \sigma_{b} \in \mathrm{O}^{+}(V) \backslash\{1,-1\}$.

(b) Let $\mathrm{d} V=\lambda K^{* 2}$. Take a basis $v_{1}, \ldots, v_{n}$ for $V$ such that $f\left(v_{i}, v_{j}\right)=0$ for all $i, j \in\{1, \ldots, n\}$ when $i+j>n+1$, and $f\left(v_{i}, v_{j}\right)+f\left(v_{i-1}, v_{j}\right)+f\left(v_{i}, v_{j-1}\right)=0$ for all $i, j \in\{2, \ldots, n\}$ (the construction will be done later). Define $\pi \in \mathrm{GL}(V)$ such that $v_{i} \pi:=v_{i}+v_{i+1}$ for $i \in\{1, \ldots, n-1\}$ and $v_{n} \pi:=v_{n}$. Then $\operatorname{mip}(\pi)=(x-1)^{n}$ and $\pi \in \mathrm{O}(V)$ as $f\left(v_{i} \pi, v_{j} \pi\right)=f\left(v_{i}, v_{j}\right)$ for all $i, j \in\{1, \ldots, n\}$ by our assumptions. Hence $\pi-\operatorname{type}(V)=2^{-}$. We will construct a basis with the above properties:

Consider a system of linear equations with unknowns $f_{i, j}$ where $i, j \in\{1, \ldots, n\}$ and $i \leq j$ :

$$
\begin{aligned}
f_{i . j}=0 & \text { if } i+j>n+1 \text { and } f_{l . n}=\lambda \\
f_{i . j}+f_{i-1 . j}+f_{i . j-1}=0 & \text { if } i, j \in\{2, \ldots, n\} \text { and } i<j \\
f_{i . i}+2 f_{i-1 . i}=0 & \text { for } i \in\{2, \ldots, n\}
\end{aligned}
$$

This system of equations has a solution: compute recursively $f_{i, j}$ for fixed $i+j$.

Now let $g$ be the symmetric bilinear form whose Gram matrix $\left(g_{i, j}\right)$ is given by $g_{i, j}:=f_{i, j}$ when $i \leq j$ and $g_{i, j}:=f_{j, i}$ when $j<i$. Then ind $(V, g)=(n-1) / 2=$ ind $(V, f)$ and $\mathrm{d}(V, g)=\lambda K^{* 2}=\mathrm{d}(V, f)$. Hence $(V, f)$ is isometric to $(V, g)$. Therefore, one can find a basis $v_{1}, \ldots, v_{n}$ for $V$ such that the Gram-matrix of $f$ associated with this basis is $G$. This basis fulfils our requirements.

Proof of (c) and (d). Take totally isotropic subspaces $U, W$ such that $V=U \oplus W$. Suppose that $q \in K[x]$ where $\operatorname{degree}(q)=n / 2, q \neq q^{*}$ and $q$ is a power of an irreducible polynomial. Let $\varphi \in \mathrm{GL}(V)$ be such that $\operatorname{mip}(\varphi)=q$. Then Lemma 4.4 supplies $\pi \in \mathrm{O}(V)$ such that $\pi_{U}=\varphi$. As $\operatorname{char}(\pi)$ is symmetric we conclude that $\operatorname{mip}(\pi)=q q^{*}$. We proved (c) and assume that $n \equiv 0 \bmod 4$. Take $\varphi \in \mathrm{GL}(U)$ such that $\operatorname{mip}(\varphi)=(x+1)^{n / 2}$. Again Lemma 4.4 supplies $\pi \in O(V)$ such that $\pi_{U}=\varphi$ and $\operatorname{mip}(\pi)=(x+1)^{n}$. Hence $\pi-\operatorname{type}(V)=1^{+}$. The same argument applies to $x-1$.

LEMMA 8.3. Let $n \geq 3$ and $\mathrm{u}(K) \leq 2$.

(a) If $n \equiv 2 \bmod 4$ then $\Omega(V)$ is not 2-reflectional.

(b) If $n$ is odd and $n \neq 9$ and $\mathrm{s}(K) \neq 1$ then $\Omega(V)$ is not 2-reflectional. 
(c) If $n \notin\{8,9\}, \mathrm{s}(K) \neq 1$ and $\mathrm{d} V=K^{* 2}$ then $\Omega(V)$ contains some $\pi$ which is not a product of two involutions in $\operatorname{ker}(\Theta)$.

PROOF OF (a). We have $V=A \oplus H_{1} \oplus H_{2} \oplus W$ where $H_{i}$ are hyperbolic planes, $W$ is a hyperbolic space such that $\operatorname{dim}(W) \equiv 0 \bmod 4$ and $\operatorname{dim}(A)=2$ where $A$ is a hyperbolic plane or an anisotropic space. Hence $\mathrm{d} A=\mathrm{d} V$. If $A$ is anisotropic or $|K| \geq 3$ then Lemma 8.2 (a) supplies $\psi \in \mathrm{O}(A)$ such that $\psi$-type $(A)=2^{*}$ or 3 . If $W \neq 0$ then Lemma 8.2 (d) supplies some $\zeta \in O(W)$ such that $\zeta$-type $(W)=1$.

Case 1: $K$ is an infinite field. Let $\lambda \in K^{*}$ be such that $\Theta(\psi)=\lambda K^{* 2}$. Select $\mu_{1} \in K^{*} \backslash\left\{1,-1, \lambda^{-1},-\lambda^{-1}\right\}$ and let $\mu_{2}:=\left(\lambda \mu_{1}\right)^{-1}$. Let $\varphi_{i} \in \mathrm{O}\left(H_{i}\right)$ be such that $\varphi_{i}$-type $\left(H_{i}\right)=3$ and $\Theta\left(\varphi_{i}\right)=\mu_{i} K^{* 2}$; cf. Lemmas 8.2 (c) and 4.7. Then $\pi:=\psi\left(\varphi_{1} \oplus \varphi_{2} \oplus \zeta \in \Omega(V)\right.$.

Case 2.1: $K$ is finite and $\mathrm{A}$ is anisotropic. Choose an irreducible quadratic monic polynomial $q \in K[x]$ such that $q(0) \cdot K^{* 2}=\Theta(\psi)$. If $\Theta(\psi) \neq K^{* 2}$ then $q \neq q^{*}$ and Lemma 8.2 (c) supplies $\varphi \in \mathrm{O}\left(H_{1} \oplus H_{2}\right)$ such that $\operatorname{mip}(\varphi)=q q^{*}$ and we obtain $\Theta(\varphi)=q(0) K^{* 2}=\Theta(\psi)$; cf. Lemma 4.7 If $\Theta(\psi)=K^{* 2}$ choose $\varphi \in$ $\mathrm{O}\left(H_{1} \oplus H_{2}\right)$ such that $\varphi$-type $\left(H_{1} \oplus H_{2}\right)=1$; cf. Lemma 8.2 (d). This choice yields $\pi:=\psi \oplus \varphi \oplus \zeta \in \Omega(V)$.

Case 2.2: $K$ is finite and $\mathrm{A}$ is a hyperbolic plane. Take an irreducible monic polynomial $q \in K[x]$ of degree 3 such that $q(0)=-1$; cf. Proposition 4.13. Now Lemma $8.2(\mathrm{c})$, provides $\varphi \in \mathrm{O}\left(A \oplus H_{1} \oplus H_{2}\right)$ such that $\operatorname{mip}(\varphi)=q q^{*}$. Then $\pi:=\varphi \oplus \zeta \in \Omega(V)$.

In each of the cases 1, 2.1 and 2.2 we conclude from Proposition 4.6 and Corollary 5.9 that $\pi$ is not a product of two involutions in $\mathrm{O}^{+}(V)$.

PROOF OF (b). Case 1: $n \equiv 3$ or $5 \bmod 8$. Let $\pi \in \Omega(V)$ be such that $\pi-\operatorname{type}(V)=$ $2^{-}$: cf. Lemma 8.2 (b). Suppose that $\rho, \sigma \in \mathrm{O}(V)$ are involutions such that $\pi=\rho \sigma$. Then Lemma 5.1 implies that

$$
\rho \in \mathrm{O}^{+}(V) \text { and } \Theta(\rho)=-K^{* 2} \text {, or } \rho \in \mathrm{O}^{-}(V) \text { and } \Theta(\rho)=-\mathrm{d} V \text {. }
$$

Case 2: $n \equiv 7 \bmod 8$. As $\mathrm{u}(K) \leq 2$ we have a decomposition $V=V_{1}(1) V_{2}(1) V_{3}$ where $\operatorname{dim}\left(V_{1}\right)=\operatorname{dim}\left(V_{2}\right)=1$ and $\operatorname{dim}\left(V_{3}\right) \equiv 5 \bmod 8$ and $\mathrm{d} V_{1}=\mathrm{d} V_{2}=\mathrm{d} V_{3}=\mathrm{d} V$. Now Lemma 8.2 provides $\pi \in \mathrm{O}(V)$ such that $V_{1} \leq \mathrm{F}(\pi)$ and $V_{2} \leq \mathrm{N}(\pi)$ and $\pi$-type $\left(V_{3}\right)=2^{+}$. Hence $\pi \in \Omega(V)$; cf. Lemma 4.7. Let $\rho, \sigma \in \mathrm{O}(V)$ be arbitrary involutions with $\pi=\rho \sigma$. Due to Proposition 4.6 we can assume that the above decomposition is invariant under $\rho$. Therefore, Lemma 5.1 implies that $(*)$ holds true. Case 3: $n \equiv 1 \bmod 8$. Then $n \geq 17$ since $n \neq 9$. Thus we find a decomposition $V=V_{1} \oplus V_{2} \oplus V_{3}$ where $\operatorname{dim}\left(V_{1}\right)=\operatorname{dim}\left(V_{2}\right)=7$ and $\operatorname{dim}\left(V_{3}\right) \equiv 3 \bmod 8$ and $\mathrm{d} V_{1}=$ $\mathrm{d} V_{2}=\mathrm{d} V_{3}=\mathrm{d} V$. Choose $\pi \in \mathrm{O}(V)$ such that $\pi-\operatorname{type}\left(V_{1}\right)=2^{-}, \pi-\operatorname{type}\left(V_{2}\right)=2^{+}$ 
and $\pi$-type $\left(V_{3}\right)=2^{+}$; cf. Lemma 8.2 (b). Again we obtain that $\pi \in \Omega(V)$ and $\left(^{*}\right)$ for arbitrary orthogonal involutions with $\pi=\rho \sigma$.

In each of the three cases we obtained in particular that arbitrary involutions $\rho, \sigma \in \mathrm{O}(V)$ with $\pi=\rho \sigma$ are not elements of $\Omega(V)$. Furthermore, under the assumption that $\mathrm{d} V=K^{* 2}, \rho$ and $\sigma$ are not elements of $\operatorname{ker}(\Theta)$.

PrOOF OF (c). Suppose that the assumptions of (c) hold true. If $n$ is odd then the claim was proved in the proof of (b). Suppose that $n$ is even.

Case 1: $n \equiv 0 \bmod 8$. As $n \neq 8$ we have $n \geq 16$. Hence we find a decomposition $V=V_{1} \oplus V_{2} \oplus V_{3}\left(V_{4}\right.$ such that $\operatorname{dim}\left(V_{1}\right)=\operatorname{dim}\left(V_{2}\right)=1, \operatorname{dim}\left(V_{3}\right)=9, \operatorname{dim}\left(V_{4}\right) \equiv$ $5 \bmod 8$ and $\mathrm{d} V_{1}=\cdots=\mathrm{d} V_{4}=K^{* 2}$. Using Lemma 8.2 we obtain $\pi \in \mathrm{O}(V)$ such that $V_{1} \subseteq \mathrm{F}(\pi), V_{2} \subseteq \mathrm{N}(\pi), \pi-\operatorname{type}\left(V_{3}\right)=2^{+}$and $\pi-\operatorname{type}\left(V_{4}\right)=2^{-}$. This choice yields in particular $\pi \in \Omega(V)$. From Proposition 4.6 and Lemma 5.1 we conclude that $\pi$ is not a product of two involutions in $\operatorname{ker}(\Theta)$.

Case 2: $n \equiv 2 \bmod 8$. Then $n \geq 10$. We find a decomposition $V=V_{1} \oplus V_{2}$ such that $\operatorname{dim}\left(V_{1}\right)=7$ and $\operatorname{dim}\left(V_{2}\right) \equiv 3 \bmod 8$ and $\mathrm{d} V_{1}=\mathrm{d} V_{2}=K^{* 2}$. Using Lemma 8.2 we obtain $\pi \in \mathrm{O}(V)$ such that $\pi$-type $\left(V_{1}\right)=2^{-}=\pi-\operatorname{type}\left(V_{2}\right)$. Again we conclude that $\pi \in \Omega$ and $\pi$ is not a product of two involutions in $\operatorname{ker}(\Theta)$.

Case 3: $n \equiv 4 \bmod 8$. As $\mathrm{d} V=K^{* 2}$ we know that $V$ is a hyperbolic space. Hence Lemma 8.2 provides $\pi \in \mathrm{O}(V)$ such that $\pi$-type $(V)=1$. So $\pi \in \Omega(V)$ and $\pi$ is not a product of two involutions in $\operatorname{ker}(\Theta)$; cf. Lemma 5.5.

Case 4: $n \equiv 6 \bmod 8$. We find a decomposition $V=V_{1} \oplus V_{2}$ such that $\operatorname{dim}\left(V_{1}\right)=1$ and $\operatorname{dim}\left(V_{2}\right) \equiv 5 \bmod 8$ and $\mathrm{d} V_{1}=\mathrm{d} V_{2}=K^{* 2}$. Using Lemma 8.2 we obtain $\pi \in O(V)$ such that $\pi-\operatorname{type}\left(V_{1}\right)=2^{-}=\pi-\operatorname{type}\left(V_{2}\right)$. We conclude that $\pi \in \Omega(V)$ and $\pi$ is not a product of two involutions in $\operatorname{ker}(\Theta)$; cf. Proposition 4.6 and Lemma 5.1.

LEMMA 8.4. Let $7 \leq|K|<\infty$.

(a) If $n=4$ and $V$ is a hyperbolic space, or $n=5$, then $\Omega(V)$ is 3-reflectional.

(b) If $n \in\{3,4,5\}$ then $\operatorname{ker}(\Theta)$ is 3-reflectional.

PROOF OF (a). First suppose that $n=4$ and $V$ is a hyperbolic space. Let $\pi \in \Omega(V)$. Case 1: $\mathrm{F}(\pi) \cup \mathrm{N}(\pi)$ contains an anisotropic vector $v$. Then Corollary 7.2 (a) implies that $\pi$ or $-\pi$ is a product of at most three involutions in $\Omega(V)$. So $\pi$ is a product of at most three involutions in $\Omega(V)$.

Case 2: $\quad V=U(1) W$ for $\pi$-modules $U, W$ where $\operatorname{dim}(U)=2$ and $\pi_{U} \in \mathrm{O}^{+}(U)$. As $\mathrm{u}(K)=2$ we find symmetries $\rho_{1}, \rho_{2} \in \mathrm{O}(U)$ and $\sigma_{1}, \sigma_{2} \in \mathrm{O}(W)$ such that $\pi_{U}=\rho_{1} \rho_{2}$, $\pi_{W}=\sigma_{1} \sigma_{2}$ and $\Theta\left(\rho_{1}\right)=\Theta\left(\sigma_{1}\right)$. Then $\pi=\left(\rho_{1} \oplus \sigma_{1}\right) \cdot\left(\rho_{2} \oplus \sigma_{2}\right)$ shows that $\pi$ is a product of two involutions in $\Omega(V)$. 
Case 3: None of the cases 1 or 2 applies. Then an orthogonal decomposition of $V$ into $\pi$-modules does not contain modules of dimension 1 or 3 (case 1). Definition 3.5 leaves the possibilities: $V$ is an orthogonally indecomposable $\pi$-module [then $\pi$-type $(V)=$ 1,3 or $2^{*}$, where in the latter case $\operatorname{mip}(\pi)$ is a square] or $V=U \oplus W$ where $U$ and $W$ are 2-dimensional orthogonally indecomposable $\pi$-modules. The second possibility does not occur: as case 2 is excluded we have $\operatorname{det}\left(\pi_{U}\right)=-1$; hence $\pi$-type $(U)=2^{+}$, in particular $\operatorname{dim}(U)$ is odd. Hence $V$ contains a 2-dimensional totally isotropic $\pi$-module $T \leq V$ which is $\pi$-cyclic unless $\pi_{r}=1_{r}$ or $-1_{r}$. (that is, when $\pi$-type $(V)=1$ ). Clearly, one can find a totally isotropic subspace $S$ such that $V=T \oplus S$ and $S \pi \neq S$. Take a basis $t_{1}, t_{2}$ for $T$ and a basis $s_{1}, s_{2}$ for $S$ such that the Gram-matrix of $f$ (associated to the basis $t_{1}, t_{2}, s_{1}, s_{2}$ of $V$ ) is $G=\left(\begin{array}{c}0 \\ E \\ E\end{array}\right)$ where $E$ denotes the $2 \times 2$-unit-matrix. If $\pi$-type $(V) \neq 1$ we can additionally assume that $t_{1} \pi=t_{2}$. Then $\pi=\left(\begin{array}{ll}P & 0 \\ x & \varrho\end{array}\right)$ where $P=\left(\begin{array}{ll}0 & 1 \\ \alpha & \beta\end{array}\right)$ for some $\alpha, \beta \in K$, respectively $P=E$ when $\pi-\operatorname{type}(V)=1$. As $\pi$ is orthogonal we have $G=\pi G \pi^{t}$. This implies that $Q=\left(P^{-1}\right)^{t}$ and $X=\left(\begin{array}{cc}0 & \gamma \\ -\gamma & 0\end{array}\right)$ where $\gamma \in K^{*}$, provided $P \notin\{E,-E\}$. As $|K| \geq 5$ we can choose $\lambda \in K^{* 2} \backslash\{-\alpha\}$. For $\delta \in K^{*}$ let $\omega:=\left(\begin{array}{cc}0 & A \\ A^{-1} & 0\end{array}\right)$ where $A:=\left(\begin{array}{ll}\delta & 0 \\ 0 & \lambda \delta\end{array}\right)$. Observe that $\omega$ is an involution and $\omega \in \operatorname{ker}(\Theta) \cap \mathrm{O}^{+}(V)=\Omega(V)$. Hence $\pi \omega \in \Omega(V)$. An elementary calculation yields that $\operatorname{char}(\pi \omega)=x^{4}+\mu x^{3}+\nu x^{2}+\mu x+1$ where $\mu=-\operatorname{trace}(X A)=\delta \gamma(\lambda-\alpha)$ and $\nu=-\left(\lambda \alpha^{-1}\right)\left((\delta \gamma \alpha)^{2}+1+\left(\alpha \lambda^{-1}\right)^{2}\right)$ provided $P \notin\{E,-E\}$, or else $\mu=0$ and $\nu=\lambda \delta^{2} \gamma^{2}-2$.

We contend that a suitable choice of $\delta$ entails that $\pi \omega$ fulfils the assumptions of case 2 ; then $\pi \omega$ is a product of two involutions in $\Omega(V)$ and $\pi$ is a product of three involutions in $\Omega(V)$.

First, let us consider the case $P \in\{E,-E\}$. As $\mathrm{u}(K) \leq 2$ and $|K| \geq 7$ we can choose $\delta \in K^{*}$ and some $u \in K^{*}$ such that $\lambda \delta^{2} \gamma^{2}+u^{2}=4$; cf. Lemma 4.11. This choice yields that $\operatorname{char}(\pi \omega)=\left(x^{2}+u x+1\right)\left(x^{2}-u x+1\right)$; hence $\operatorname{char}(\pi \omega)$ is a product of two symmetric quadratic polynomials where the first is prime to the second one. Therefore, $\pi \omega$ fulfils the assumptions of case 2 and we have finished.

Secondly, assume that $P \notin\{E,-E\}$. Take $\kappa, \eta \in K^{*}$ such that $\kappa^{2}-\eta^{2}=$ $-\left(\lambda \alpha^{-1}\right)\left(1+\alpha \lambda^{-1}\right)^{2}$ [this is possible as a hyperbolic plane is universal and, due to $|K| \geq 7$, one has at least 3 linearly independent solutions $(\kappa, \eta) \in K \times K$; cf. Lemma 4.11]. Take $\delta:=2 \kappa(\gamma(\alpha+\lambda))^{-1}$. Then $\operatorname{char}(\pi \omega)=\left(x^{2}+u x+1\right)\left(x^{2}+\right.$ $w x+1)$ where $u:=\eta+\mu / 2$ and $w:=\mu-u$. Furthermore, $u \neq w$ (as $\eta \neq 0$ ). So $\operatorname{char}(\pi \omega)$ is a product of two symmetric quadratic polynomials where the first is prime to the second one. Hence $\pi \omega$ fulfils the assumptions of case 2 and we have finished.

Now we consider $n=5$.

Case 1: $F(\pi)$ contains an anisotropic vector. Then the assertion follows immediately from the previous one (when $v^{\perp}$ is a 4-dimensional hyperbolic space) or Corollary 7.2 
(b).

Case 2: $\quad V$ contains a 2-dimensional regular $\pi$-module $U$ such that $\pi_{U} \in \mathrm{O}^{+}(U)$. Then $\pi_{U^{\perp}}=\sigma_{1} \sigma_{2}$ where $\sigma_{i}$ are orthogonal involutions. As $\mathrm{u}(K) \leq 2$ we have $\pi_{U}=$ $\rho_{1} \rho_{2}$ where $\rho_{i}$ are symmetries and $\Theta\left(\rho_{1}\right)=\Theta\left(\sigma_{1}\right)$. Hence $\pi=\left(\rho_{1} \oplus \sigma_{1}\right) \cdot\left(\rho_{2} \oplus \sigma_{2}\right)$ proves that $\pi$ is a product of two involutions in $\Omega(V)$.

Case 3: Neither case 1 nor case 2. Then $V$ is an orthogonally indecomposable $\pi$ module, and, since $\operatorname{dim}(V)$ is odd and $\pi \in \mathrm{O}^{+}(V), \pi-\operatorname{type}(V)=2^{-}$. Let $\mathrm{d} V=\mu K^{* 2}$. Select $\lambda$ such that $\mathrm{d} V \neq \lambda K^{* 2}$. Then $4 \lambda-\mu \neq 0$. Clearly, $\operatorname{dim}(\operatorname{rad}(\mathrm{B}(\pi)))=1$ and $\operatorname{dim}\left(\operatorname{rad}\left(B^{2}(\pi)\right)\right)=2$. Hence we find isotropic vectors $y, z$ such that $y \in$ $\mathrm{B}(\pi) \backslash \mathrm{B}^{2}(\pi)$ and $z \in \mathrm{B}^{2}(\pi)$ and $\langle y, z\rangle$ is a hyperbolic plane. In particular, one has some $u \in \mathrm{B}(\pi) \backslash \mathrm{B}^{2}(\pi)$ such that $q(u)=4 \lambda-\mu$. Let $v \in V$ be such that $v(\pi-1)=u$ and $w \in \mathrm{F}(\pi)=\operatorname{rad}(\mathrm{B}(\pi))$ with $w \neq 0$. Then $v \notin \mathrm{B}(\pi)$; hence $\langle v, w\rangle$ is a hyperbolic plane. Therefore, $q(z)=\lambda$ for some $z=v+\beta w$ where $\beta \in K$. We have $z(\pi-1)=u$ and $q(z(\pi+1))=2 q(z)+2 f(z, z \pi)=4 q(z)-q(z(\pi-1))=4 \lambda-q(u)=\mu \in \mathrm{d} V$. Let $\omega:=-\sigma_{z(\pi+1)}$. Then $\operatorname{det}(\omega)=1$ and $\Theta(\omega)=q(z(\pi+1)) \mathrm{d} V=K^{* 2}$. We have $z \in \mathrm{F}(\pi \omega)$ and $\mathrm{d}\left(z^{\perp}\right)=q(z) \mathrm{d} V \neq K^{* 2}$. Thus $\pi \omega$ is a product of two involutions of $\Omega(V)$ (apply Corollary 7.2 (b) to the restriction of $\pi \omega$ to $z^{\downarrow}$ ). We have proved that $\pi$ is a product of three involutions in $\Omega(V)$.

PROOF OF (b). Let $n \in\{3,4,5\}$ and $\pi \in \operatorname{ker}(\Theta)$. We want to prove that $\pi$ is a product of three involutions in $\operatorname{ker}(\Theta)$. If $\pi \in \Omega(V)$ then Corollary 7.2 or part (a) prove the assertion. Hence we can assume that $\pi \notin \Omega(V)$. Take orthogonal involutions $\rho, \sigma$ such that $\pi=\rho \sigma$. If $\mathrm{d} V \neq K^{* 2}$ then $\rho \in \operatorname{ker}(\Theta)$ or $-\rho \in \operatorname{ker}(\Theta)$ and it follows that $\pi$ is a product of two involutions of $\operatorname{ker}(\Theta)$. So let $\mathrm{d} V=K^{* 2}$. If $n \in\{3,5\}$ then $-\pi \in \Omega(V)$, and we have seen that $-\pi$ is a product of three involutions in $\Omega(V)$; hence $\pi$ is a product of three involutions in $\operatorname{ker}(\Theta)$. Let $n=4$. As $\operatorname{det}(\pi)=-1$ an orthogonal decomposition of $V$ into orthogonally indecomposable $\pi$-modules contains at least one module of type $2^{+}$. All $\pi$-modules but those of type $2^{+}$or $2^{-}$have even dimension. Hence $V$ contains an anisotropic $t \in N(\pi) \cup \mathrm{F}(\pi)$. From our result for $n=3$ we know that $-\pi$ or $\pi$ is a product of three involutions in $\operatorname{ker}(\Theta)$. So $\pi$ is a product of three involutions in $\operatorname{ker}(\Theta)$.

THEOREM 8.5. Let $K$ be finite and $n \geq 3$. If $|K|=3$ suppose additionally that $n \geq 6$ or $\left[n=4\right.$ and $\left.\mathrm{d} V=-K^{* 2}\right]$. Then $\Omega(V)$ is 3-reflectional. $\Omega(V)$ is 2reflectional if and only if

(i) $\mathrm{s}(K)=1$ and $n \not \equiv 2 \bmod 4$, or

(ii) $n \equiv 0 \bmod 4$ and $\mathrm{d} V=-K^{* 2}$, or

(iii) $n \in\{8,9\}$.

PROOF. First we prove that $\Omega(V)$ is 2 -reflectional provided one of the assumptions 
(i), (ii), (iii) holds true. Under each of these assumptions we get involutions $\rho, \sigma \in$ $\mathrm{O}^{+}(V)$ such that $\pi=\rho \sigma$ (first statement in Section 8). Consider a decomposition $\mathscr{D}$ of $V$ into orthogonally indecomposable $\pi$-modules which are simultaneously $\rho$ modules; cf. Proposition 4.6.

(+) The assertion is correct if $\mathscr{D}$ contains a summand $X$ such that $\operatorname{dim}(X) \equiv$ $2 \bmod 4$, or $\pi-\operatorname{type}(X)=2^{*}$ and $\operatorname{mip}\left(\left.\pi\right|_{X}\right)$ is not a square in $K[x]$.

PROOF OF (t). An onhogonal summand whose dimension is congruent $2 \bmod 4$ has type $2^{*}$ or 3 . So in thw case Lemma 5.8 yields the assertion. Now suppose that $\mathscr{D}$ contains an orthogonal summand $X$ of type $2^{*}$ such that $\operatorname{mip}\left(\left.\pi\right|_{X}\right)$ is not a square in $K[x]$. We may assume that $\operatorname{dim}(X) \equiv 0 \bmod 4$ (else the previous argument applies). From Lemma 4.8 " tollim, that $X$ is not a hyperbolic space. Hence $\mathrm{d} X \neq K^{* 2}$. We conclude that $\left(\Theta\left(\rho_{X}\right) \neq+-\rho_{1}\right)$ and $\operatorname{dim}\left(\mathrm{B}\left(\rho_{X}\right)\right)=\operatorname{dim}\left(\mathrm{B}\left(-\rho_{X}\right)\right)$; cf. Corollary 5.9. Hence $\rho \in \Omega\left(V^{\prime}\right.$ ) or $\prime^{\prime}=-\rho$. D $\rho_{X^{+}} \in \Omega(V)$. So $\pi=\rho \sigma$ or $\pi=\rho^{\prime} \sigma^{\prime}$ is a product of two involution in $\Omega .1, W_{c}$ have proved statement $(+)$.

In what follow w w siume that the assumptions of $(+)$ are not fulfilled. Hence every orthogonall! inde.urmpnashle $\pi$-module $X$ in $V$ satisfies:

$(++)$ if $\pi \cdot t$ $\pi$-type $(X)=3 \operatorname{then} \operatorname{din} 11=0 \bmod 4$.

(i) Let $s\left(K^{\prime}\right)=1$ ain $r=2 \bmod 4$. Statements $(++), 5.1,5.2,5.3,5.5$ and 5.7 provide orthogonal inwiumun,, , w such that $\pi=\eta \omega$ and $\mathrm{B}(\eta)$ is a hyperbolic space. Since $s(K)=1 \|$ thllinu . that $H(\eta)=K^{* 2}$ and $\pi$ is a product of two involutions in $\Omega(V)$.

(ii) Let $n \equiv 0 \mathrm{mkN}+$ and $\mathrm{dl}=-K^{* 2}$. From case (i) we may assume that $\mathrm{s}(K)=$ 2. As $\mid K^{*} / K^{* 2}:=2$ and $\mathrm{dl}=-K^{* 2}$ we have $\rho, \sigma \in \Omega(V)$ or $-\rho,-\sigma \in \Omega(V)$.

(iii) Now let $n \in\{8.9\}$ Let $U^{\prime}:=\mathrm{F}^{\infty}\left(\pi^{2}\right)$ and $W:=\mathrm{B}^{\infty}\left(\pi^{2}\right)$. Then $U, W$ are $\pi$-modules and $V=L U$. An orthogonal decomposition of $U$ into orthogonally indecomposable $\pi$-modules contains only modules of type $1,2^{-}$or $2^{+}$; and $W$ contains only those of the remaining types $2^{*}$ and 3 (where (++) imposes additional restrictions). Therefore, $\operatorname{dim}(W) \in\{0.4 .8\}$.

If $\operatorname{dim}(W)=8$ then Lemma 5.3 (for type $2^{*}$ and $m$ even) or Lemma 5.7(i) supply involutions $\rho_{1}, \sigma_{1} \in \mathrm{O}^{+}(W)$ such that $\pi_{W}=\rho_{1} \sigma_{1}$ and $\Theta\left(\rho_{1}\right)=K^{* 2}$. Clearly, this yields the assertion.

Now let $\operatorname{dim}(W)=4$. Then Lemmas 5.3 or 5.7 (i) supply involutions $\rho_{1}, \sigma_{1} \in$ $\mathrm{O}^{+}(W)$ such that $\pi_{W}=\rho_{1} \sigma_{1}$ and $\Theta\left(\rho_{1}\right)=-K^{* 2}$. Consider the possible orthogonal decompositions of $U$ (taking into account $(++)$ ). Using 5.1, 5.2 and 5.5 we obtain 
that $\pi_{U}=\rho_{2} \sigma_{2}$ for involutions $\rho_{2}, \sigma_{2} \in \mathrm{O}^{+}(U)$ such that $\Theta\left(\rho_{2}\right)=-K^{* 2}$. As $\pi=\left(\rho_{1} \oplus \rho_{2}\right)\left(\sigma_{1} \oplus \sigma_{2}\right)$ and $\rho_{1} \oplus \rho_{2} \in \Omega(V)$ the claim is proved.

Finally, let $\operatorname{dim}(W)=0$; hence $V=U$.

We study the possible orthogonal decompositions of $\mathrm{V}$ into orthogonally indecomposable $\pi$-modules. The claim follows immediately from 5.1, 5.2 and 5.5 with one exception that deserves special analysis, namely the following one: $n=9$ and $V=X \oplus Y(1) Z$ where $X, Y, Z$ are 3-dimensional and of $\pi$ type $2^{+}$or $2^{-}$. We may assume that $\pi$-type $(X)=2^{+}=\pi-\operatorname{type}(Y)$ and $\pi$-type $(Z)=2^{-}$. We claim that $X \oplus Y=X^{\prime} \oplus Y^{\prime}$ where $\pi$-type $\left(X^{\prime}\right)=2^{+}=\pi-\operatorname{type}\left(Y^{\prime}\right)$ and $\mathrm{d} Y^{\prime}=-\mathrm{d} Z$.

PROOF OF THIS ClAIM. Let $A:=(X \oplus Y)(\pi+1)$. Then $\operatorname{dim}(A)=4$ and $A$ contains a 2-dimensional regular subspace. As $\mathrm{u}(K) \leq 2$ we find $a \in A$ such that $q(a) \in \mathrm{d} Z$. Let $v \in X(1) Y$ be such that $v(\pi+1)=a$. Then $\langle v, a, a(\pi+1)\rangle$ is a basis for the $\pi$-cyclic subspace $\langle v\rangle_{\pi}$ and the assigned Gram-matrix is (cf. Lemma 2.10)

$$
\left(\begin{array}{ccc}
q(v) & f(v, a) & f(v, a(\pi+1)) \\
f(v, a) & q(a) & 0 \\
f(v, a(\pi+1) & 0 & 0
\end{array}\right) .
$$

Therefore, $\mathrm{d}\langle v\rangle_{\pi}=-\mathrm{d} Z$. Take $Y^{\prime}:=\langle v\rangle_{\pi}$ and $X^{\prime}:=(X \oplus Y) \cap X^{\perp}$. Then $X^{\prime}, Y^{\prime}$ fulfil the requested properties.

Now $\pi_{X^{\prime}}=\sigma_{1} \sigma_{2}$ for involutions $\sigma_{1}, \sigma_{2} \in \operatorname{SO}(V)$ with $\Theta\left(\sigma_{1}\right)=-K^{* 2}$ by 5.1 and 5.2. Also 5.1 and 5.2 yield that $\pi_{Y^{\prime}}=\rho_{1} \rho_{2}$ for involutions $\rho_{1}, \rho_{2} \in \mathrm{O}^{-}\left(Y^{\prime}\right)$ with $\Theta\left(\rho_{1}\right)=-\mathrm{d} Y^{\prime}=\mathrm{d} Z$, and $\pi_{Z}=\omega_{1} \omega_{2}$ for involutions $\omega_{1}, \omega_{2} \in \mathrm{O}^{\prime}(Z)$ with $\Theta\left(\omega_{1}\right)=-\mathbf{d} Z$. The identity $\pi=\left(\sigma_{1} \oplus \rho_{2} \oplus \omega_{1}\right)\left(\sigma_{2} \oplus \rho_{2}(1) \omega_{2}\right)$ proves the assertion.

If none of the cases (i), (ii) or (iii) is present then $\Omega(V)$ is not 2-reflectional; cf. Lemma 8.3. If $n \geq 6$ then Theorem 7.5 yields 3 -reflectionality of $\Omega(V)$. If $n \leq 5$ then Corollary 7.2 and Lemma 8.4 yield 3-reflectionality of $\Omega(V)$ provided $K \neq \mathrm{GF} 5$. The case $K=\mathrm{GF} 5$ is covered by (i) as $\mathrm{s}(\mathrm{GF} 5)=1$.

THEOREM 8.6. Let $K$ be finite and $n \geq 3$. If $|K|=3$ suppose additionally that $n \geq 6$ or $\mathrm{d} V=-K^{* 2}$. Then $\operatorname{ker}(\Theta)$ is 3-reflectional. Furthermore, $\operatorname{ker}(\Theta)$ is 2-reflectional if and only if

(i) $\mathrm{s}(K)=1$, or

(ii) $\mathrm{d} V=-K^{* 2}$, or

(iii) $n \in\{8,9\}$.

The proof is an analogue of the previous one. 
LEMMA 8.7. Let $K$ be a euclidean field, $n \geq 4$ and ind $(V) \neq 2$. Let $\pi=\rho \sigma$ where $\rho, \sigma$ are orthogonal involutions and $\operatorname{dim}(\mathrm{B}(\rho)) \leq 2$. Then $\pi=\omega \delta$ for involutions $\omega \in \Omega(V)$ and $\delta \in \mathrm{O}(V)$.

PROOF. Choose a regular subspace $U$ of $V$ such that $\left.\pi\right|_{U}$ is an involution and $\operatorname{dim}(U)$ is maximal. Then $U \leq \mathrm{F}\left(\pi^{2}\right)$, hence $\mathrm{B}\left(\pi^{2}\right) \leq U^{\perp}$.

We claim that $\pi_{U^{\perp}}=\rho^{\prime} \sigma^{\prime}$ for involutions $\rho^{\prime}, \sigma^{\prime} \in \mathrm{O}\left(U^{\perp}\right)$ such that one of the following additional statements holds true:

(i) $\rho^{\prime} \in \Omega\left(U^{\perp}\right)$,

(ii) $\rho^{\prime} \in \mathrm{O}^{-}\left(U^{\perp}\right)$ and $q(y) \in \Theta\left(\rho^{\prime}\right)$ for some $y \in U$,

(iii) $\rho^{\prime} \in \mathrm{O}^{+}\left(U^{\perp}\right)$ and $\Theta\left(\rho^{\prime}\right)=-K^{* 2}$ and $U$ is isotropic.

Before we will prove this claim, let us show that the assertion is indeed a consequence.

If (i) is true let $\omega:=\rho^{\prime}(1) 1_{U}$ and $\delta:=\sigma^{\prime}(1) \pi_{U}$. Now suppose that (ii) is given. As $K$ is a euclidean field we can assume that $y \in \mathrm{B}\left(\pi_{U}\right) \cup \mathrm{F}\left(\pi_{U}\right)$. Let $\omega:=\rho^{\prime} \oplus \sigma_{y}$ and $\delta:=\sigma^{\prime}(1) \sigma_{y} \pi_{U}$ where $\sigma_{y}$ denotes the symmetry whose negative space is $\langle y\rangle$.

Finally, suppose that (iii) is valid. As $U=\mathrm{B}\left(\pi_{U}\right) \oplus \mathrm{F}\left(\pi_{U}\right)$ and $U$ is isotropic and $K$ is a euclidean field one has a hyperbolic plane $H$ in $U$ such that $H=$ $\left(H \cap \mathrm{B}\left(\pi_{U}\right)\right) \oplus\left(H \cap \mathrm{F}\left(\pi_{U}\right)\right)$. Let $\kappa$ denote the involution in $\mathrm{O}(U)$ with negative space $H, \omega:=\rho^{\prime}(1)$ and $\delta:=\sigma^{\prime}\left(1 \kappa \pi_{U}\right.$. In each of the three cases we defined involutions $\omega, \delta \in \Omega(V)$ satisfying $\pi=\omega \delta$.

Now let us prove the above claim. If $\pi$ is an involution then (i) is valid as $U=V$. So we can assume that $\pi$ is not an involution, hence $\operatorname{dim}\left(U^{\perp}\right) \geq 2$.

Case 1: $\pi=\alpha \beta$ where $\alpha$ is a symmetry and $\beta$ is an orthogonal involution. Then $\pi^{2}$ is a product of two symmetries; hence $\operatorname{dim}\left(B\left(\pi^{2}\right)\right) \leq 2$ and $B\left(\pi^{2}\right)$ is not totally isotropic. This yields that $\operatorname{dim}(U) \geq n-3$ and $\operatorname{dim}\left(U^{\perp}\right) \leq 3$. Due to Corollary 5.10 we have $\pi_{U^{\perp}}=\rho^{\prime} \sigma^{\prime}$ where $\rho^{\prime} \in \mathrm{O}^{-}\left(U^{\perp}\right)$ and $\sigma^{\prime} \in \mathrm{O}\left(U^{\perp}\right)$ are involutions. If some $y \in U$ satisfies $q(y) \in \Theta\left(\rho^{\prime}\right)$ we arrive at (ii): Otherwise $U$ is anisotropic. We may assume that $\Theta\left(\rho^{\prime}\right)=K^{* 2}$ and $\operatorname{sgn}(U)=(0, \operatorname{dim}(U))$. If $U^{\perp}$ is a hyperbolic plane, $4.9,5.8$ and 5.9 provide $\rho^{\prime \prime}, \sigma^{\prime \prime} \in \mathrm{O}^{-}\left(U^{\perp}\right)$ such that $\pi_{U^{\perp}}=\rho^{\prime \prime} \sigma^{\prime \prime}$ and $\Theta\left(\rho^{\prime \prime}\right)=-K^{* 2}$; so (ii) is valid. If $U^{\perp}$ is a 2-dimensional anisotropic space it follows that $\operatorname{sgn}\left(U^{\perp}\right)=(2,0)$ [since $\Theta\left(\rho^{\prime}\right)=K^{* 2}$ ]. If $\operatorname{dim}\left(U^{\perp}\right)=3$ then $U^{\perp}$ is an orthogonally indecomposable $\pi^{2}$-module, and $\left(\pi^{2}\right)$ - type $\left(U^{\perp}\right)=2^{-}$[as $\operatorname{dim}\left(\mathrm{B}\left(\pi_{U^{\perp}}^{2}\right) \leq 2\right]$; in particular, $U^{\perp}$ is isotropic. Since $\mathrm{d}\left(U^{\perp}\right)=-K^{* 2}\left[\operatorname{as} \mathrm{d}\left(U^{\perp}\right)=-\pi_{U^{\perp}}^{2}=\left(-\rho^{\prime}\right) \rho^{\prime \sigma^{\prime}}\right.$ and $\Theta\left(\rho^{\prime}\right)=K^{* 2}$ by 5.1], it follows that $\operatorname{sgn}\left(U^{\perp}\right)=(2,1)$. Thus we obtained in both of the previous cases that ind $(V)=2$, contrary to our assumptions.

Case 2: Case 1 does not apply. Then $\operatorname{dim}(\mathrm{B}(\rho))=2$ and $\operatorname{dim}\left(U^{\perp}\right) \geq 4$ [indeed: If $\operatorname{dim}\left(U^{\perp}\right) \leq 3$ then $\pi_{U^{\perp}}$ is a product of a symmetry and an involution in $\mathrm{O}\left(U^{\perp}\right)$ by Corollary 5.10, hence $\pi$ is a product of a symmetry and an orthogonal involution and 
we arrive at case 1]. As $\pi^{2}=\rho \rho^{\sigma}$ we have $\operatorname{dim}\left(\mathrm{B}\left(\pi^{2}\right)\right) \leq 4$ and $\operatorname{dim}\left(\operatorname{rad}\left(\mathrm{B}\left(\pi^{2}\right)\right)\right) \leq 2$. Hence $4 \leq \operatorname{dim}\left(U^{\perp}\right) \leq 6$. Due to Proposition 4.6 we can assume that $\mathrm{B}(\rho) \leq U^{\perp}$. If $\rho \in \Omega(V)$ then (i) holds true; if $U$ is isotropic and $\rho \notin \Omega(V)$ then (iii) applies (with $\rho^{\prime}:=\left.\rho\right|_{U^{-}}$and $\left.\sigma^{\prime}:=\left.\sigma\right|_{U^{+}}\right)$.

So we assume $U$ is anisotropic and $\rho \notin \Omega(V)$; hence $\mathrm{B}(\rho)$ is a hyperbolic plane.

Suppose that $\operatorname{sgn}(U)=(0, \operatorname{dim}(U))$. If $\operatorname{dim}\left(U^{\perp}\right)=6$ then $\operatorname{dim}\left(\mathrm{B}\left(\pi^{2}\right)\right)=4$ and $\operatorname{dim}\left(\operatorname{rad}\left(B\left(\pi^{2}\right)\right)\right)=2$. Hence $U^{\perp}$ is the orthogonal sum of two 3-dimensional $\pi^{2}$ modules of type $2^{-}$. This implies that $U^{\perp}=X \oplus Y$ where $X$ and $Y$ are 3-dimensional $\pi$-modules and $\pi$-type $(X)=2^{-}$or $2^{+}$; the same holds true for $Y$. Therefore, 5.1 and 5.2 yield that $\pi_{U^{\perp}}=\rho^{\prime} \sigma^{\prime}$ where $\rho^{\prime}, \sigma^{\prime} \in \mathrm{O}\left(U^{\perp}\right)$ are involutions and $\mathrm{B}\left(\rho^{\prime}\right)$ is a 4-dimensional hyperbolic space. Thus (i) is fulfilled.

Now suppose that $\operatorname{dim}\left(U^{\perp}\right)=5$. Then $U^{\perp}$ is an indecomposable $\pi^{2}$-module of !pe $\pi^{2}-\operatorname{type}\left(U^{\perp}\right)=2^{-}$, or $U^{\perp}=X \oplus Y$ where $\pi^{2}$ - type $(X)=2^{-}$and $\operatorname{dim}(X)=3$.

We discuss the first possibility. This yields that $\operatorname{sgn}\left(U^{\perp}\right)=(3,2)$ and $n \neq 5$ [as ind $\left(l^{\prime}\right) \neq 2$ ]. In particular, $\mathrm{d} U^{\perp}=K^{* 2}$. Thus 5.1 and 5.2 yield that $\pi_{U^{\perp}}=\rho^{\prime} \sigma^{\prime}$ where $\prime^{\prime} \cdot \sigma^{\prime} \in \mathrm{O}\left(U^{\perp}\right)$ are involutions and $\operatorname{dim}\left(\mathrm{B}\left(\rho^{\prime}\right)\right)=3$ and $\Theta\left(\rho^{\prime}\right)=-K^{* 2}$. Furthermore [a $l^{\prime} \neq 0$ and $\left.\operatorname{sgn}(U)=(0, \operatorname{dim}(U))\right]$ we have some $y \in U$ such that $q(y)=-1$. Hence we have arrived at (ii).

Nou we study the second possibility. If $Y$ is a hyperbolic space then Lemmas 5.8 and 4.9 yield that $\pi_{U^{\perp}}=\rho^{\prime} \sigma^{\prime}$ where $\rho^{\prime}, \sigma^{\prime} \in \mathrm{O}\left(U^{\perp}\right)$ are involutions and $\rho^{\prime} \in \Omega\left(U^{\perp}\right)$; hence (i) applies. So assume that $Y$ is anisotropic.

I ung $\operatorname{sgn}(U)=(0, \operatorname{dim}(U))$ and ind $(V) \neq 2$ we see that three cases may occur:

$$
\begin{aligned}
& \text { 1) } \operatorname{gn}(Y)=(0,2) \text { and } \operatorname{sgn}(X)=(1,2) \text {, or } \\
& \text { 1h. } \operatorname{gn}(Y)=(2,0) \text { and } \operatorname{sgn}(X)=(2,1) \text {, or } \\
& \text { ‘. } \operatorname{gn}(Y)=(2,0) \text { and } \operatorname{sgn}(X)=(1,2) \text { and } n \neq 5 .
\end{aligned}
$$

Clearly $\pi_{Y}$ is a product of two positive symmetries in case (b) and (c) and two negative symmetries in case (a). Thus in case (a) and (b) 5.1 and 5.2 yield that $\pi_{1}=\rho^{\prime} \sigma^{\prime}$ where $\rho^{\prime}, \sigma^{\prime} \in \mathrm{O}\left(U^{\perp}\right)$ are involutions and $\rho^{\prime} \in \Omega(V)$, hence (i) holds true. Let us consider (c). Then we obtain from 5.1 and 5.2 that $\pi_{U^{\perp}}=\rho^{\prime} \sigma^{\prime}$ where $\rho^{\prime} . \sigma^{\prime} \in \mathrm{O}\left(U^{\perp}\right)$ are involutions such that $\operatorname{dim}\left(\mathrm{B}\left(\rho^{\prime}\right)\right)=3$ and $\Theta\left(\rho^{\prime}\right)=-K^{* 2}$; hence (ii) is fulfilled.

Finally, suppose that $\operatorname{dim}\left(U^{\perp}\right) \leq 4$. Then $\operatorname{dim}\left(U^{\perp}\right)=4$ (by assumptions in case 2 ). As $\mathrm{B}(\rho)$ is a hyperbolic plane it follows that ind $\left(U^{\perp}\right) \geq 1$; furthermore, $\operatorname{ind}\left(U^{\perp}\right) \neq 2$ lor else ind $(V)=2$ ]. Hence ind $\left(U^{\perp}\right)=1$ and therefore $\mathrm{d} U^{\perp}=-K^{* 2}$. We conclude that $-\left.\rho\right|_{U^{\perp}} \in \Omega\left(U^{\perp}\right)$ and $\pi_{U^{\perp}}=\left(-\left.\rho\right|_{U^{+}}\right)\left(-\left.\sigma\right|_{U^{\perp}}\right)$; so (i) is valid.

THEOREM 8.8. Let $K$ be a euclidean field, $n \geq 3$ and ind $(V) \neq 2$. Then $\Omega(V)$ and also $\operatorname{ker}(\Theta)$ are 3-reflectional. 
PROOF. First consider $\Omega(V)$. If $n=3$ and ind $(V)=1$ then Corollary 7.2 yields the assertion. If $n=3$ and $V$ is anisotropic then each element of $\Omega(V)$ is a product of two involutions in $\mathrm{O}^{+}(V)=\Omega(V)$; cf. Corollary 5.10.

Now let $n \geq 4$ and $\pi \in \Omega(V)$. We have involutions $\rho, \sigma \in \mathrm{O}(V)$ such that $\pi=\rho \sigma$. Take an orthogonal involution $\omega$ such that $\mathrm{B}(\omega) \leq \mathrm{B}(\rho), \omega \rho \in \Omega(V)$ and $\operatorname{dim}(\mathrm{B}(\omega))$ is as small as possible. Clearly, $\operatorname{dim}(\mathrm{B}(\omega)) \leq 2$ as $K$ is a euclidean field. Let $\eta:=(\omega \rho) \pi=\omega \sigma$. From Lemma 8.7 we obtain that $\eta$ is a product of two involutions in $\Omega(V)$; hence $\pi$ is a product of three involutions in $\Omega(V)$.

Now we tum to $\operatorname{ker}(\Theta)$. Let $\pi \in \operatorname{ker}(\Theta)$. First, assume that $n=3$. By our result on $\Omega(V)$ we may assume that $\pi \notin \Omega(V)$. If $\mathrm{d} V=K^{* 2}$ (hence $-1_{V} \in \operatorname{ker}(\Theta)$ ) then $-\pi \in \Omega(V)$ and again the result on $\Omega(V)$ proves the claim. So let $\mathrm{d} V=-K^{* 2}$. We have involutions $\rho, \sigma \in \mathrm{O}(V)$ such that $\pi=\rho \sigma=(-\rho)(-\sigma)$ and $\rho, \sigma \in \operatorname{ker}(\Theta)$ or $-\rho,-\sigma \in \operatorname{ker}(\Theta)$.

Now let $n \geq 4$. Take involutions $\rho, \sigma \in \mathrm{O}(V)$ such that $\pi=\rho \sigma$. If $\rho \in \operatorname{ker}(\Theta)$ we have finished. So let $\Theta(\rho)=-K^{* 2}$. Then one has a symmetry $\rho^{\prime} \in \mathrm{O}(V)$ such that $\mathbf{B}\left(\rho^{\prime}\right) \leq \mathbf{B}(\rho)$ and $\Theta\left(\rho^{\prime}\right)=-K^{* 2}$. Hence $\rho \rho^{\prime} \in \operatorname{ker}(\Theta)$ is an involution. Furthermore, $\rho^{\prime} \sigma$ is a product of two involutions in $\operatorname{ker}(\Theta)$; cf. Lemma 8.7. We have proved that $\pi=\left(\rho \rho^{\prime}\right) \rho^{\prime} \sigma$ is a product of three involutions in $\operatorname{ker}(\Theta)$.

\section{Commutators in orthogonal groups}

Let $G$ be a group. A commutator (in elements of $G$ ) is an element of the form $\alpha \beta \alpha^{-1} \beta^{-1}$ where $\alpha, \beta \in G$. The subgroup generated by the set of all commutators is the commutator subgroup $G^{\prime}$ of $G$. Each $\pi \in G^{\prime}$ is a product of commutators. Let $\operatorname{cl}_{G}(\pi)$ denote the minimal number of factors in such a product, and $\operatorname{cl}(G):=$ $\max \left\{\operatorname{cl}_{G}(\pi) \mid \pi \in G^{\prime}\right\} \in \mathbb{N} \cup\{\infty\}$. O. Ore conjectured: every element of a finite simple nonabelian group is a commutator. This was proved for PSL $(V)$ by Thompson and for projective symplectic groups $\operatorname{PSP}(V)$ by Nielsen (cf. [13]). We want to study the problem when $G=\Omega(V)$ is the commutator subgroup of an orthogonal group. If the field is algebraically closed it is known that $\operatorname{cl}(\Omega(V))=1$; cf. [15]. Our approach essentially treats fields with $u$-invariant $u(K) \leq 2$ and the reals $\mathbb{R}$. We write $\operatorname{cl}(\pi):=\operatorname{cl}_{\Omega(V)}(\pi)$.

If $n \leq 4$ then the isomorphisms of Lemma 4.2 and R. C. Thompson's result solve our problem:

LEMMA 9.1. Let $[n=3$ and $|K| \neq 3$ ] or $n=4$. Let $\operatorname{ind}(V)=1$. Then $\operatorname{cl}(\Omega(V))=1$.

If $[|K|=3$ and $n=3]$ or $[n=4$ and ind $(V)=2]$ then the commutator subgroup 
of $\Omega(V)$ is a proper subgroup of $\Omega(V)$; cf. [17].

LEMMA 9.2. Let $\mathrm{u}(K) \leq 2$ or $K=\mathbb{R}$, and $n \geq 3$. If $U, W$ are isometric regular subspaces of $V$ then $U \alpha=W$ for some $\alpha \in \Omega(V)$.

PROOF. Witt's theorem supplies $\beta \in \mathrm{O}(V)$ such that $U \beta=W$.

Case 1: $\beta \in \mathrm{O}^{-}(V)$. If $\mathrm{u}(K) \leq 2$ then (as $\operatorname{dim}(U) \geq 2$ or $\operatorname{dim}\left(U^{\perp}\right) \geq 2$ ) some $v \in U \cup U^{\perp}$ fulfils $q(v) \in \Theta(\beta)$. If $K=\mathbb{R}$ then some $v \in V$ satisfies $q(v) \in \Theta(\beta)$ (or else each symmetry has spinorial norm $\neq \Theta(\beta)$, and $\beta$ is a product of an odd number of such symmetries; this is impossible). As $V=U\left(1 U^{\perp}\right.$ we may again assume that $v \in U \cup U^{\perp}$. Now $\alpha:=\sigma_{v} \beta$ (where $\sigma_{v}$ denotes the symmetry with negative space $\langle v\rangle)$ satisfies the requirements.

Case 2: $\beta \in \mathrm{O}^{+}(V)$. We can assume that $\beta \notin \Omega(V)$ and $\operatorname{dim}(U) \geq \operatorname{dim}\left(U^{\perp}\right)$ (or else exchange $U$ and $\left.U^{\perp}\right)$. Then $\operatorname{dim}(U) \geq 2$. First, suppose that $\mathrm{u}(K) \leq 2$. If $U^{\perp} \neq 0$ select an anisotropic $v \in U^{\perp}$; otherwise an anisotropic $v \in U$. Take $y \in v^{\perp} \cap U$ such that $q(y) \in q(v) \Theta(\beta)$ and let $\kappa \in \mathrm{O}(V)$ denote the involution whose negative space is $\langle v, y\rangle$. Now consider the case $K=\mathbb{B}$. Then ind $(V) \neq 0$ as $\beta \notin \Omega(V)$ and $\beta$ is a product of an even number of symmetries. Hence one can find a hyperbolic plane $H$ such that $H=(H \cap U) \oplus\left(H \cap U^{\perp}\right)$. Let $\kappa$ be the orthogonal involution whose negative space is $H$. In both cases $\alpha:=\kappa \beta$ satisfies the requirements.

COROLlARY 9.3. Let $n \geq 3$ and let $\rho, \sigma \in \Omega(V)$ be involutions such that $\operatorname{dim}(\mathrm{B}(\rho))=\operatorname{dim}(\mathrm{B}(\sigma))$.

(a) If $\mathrm{u}(K) \leq 2$ then $\operatorname{cl}(\rho \sigma)=1$.

(b) If $K=\mathbb{R}$ and $\operatorname{sgn}(\mathrm{B}(\rho)))=\operatorname{sgn}(\mathrm{B}(\sigma)))$ then $\operatorname{cl}(\rho \sigma)=1$.

PROOF. 2.3 yields that $\mathrm{B}(\rho)$ is isometric to $\mathrm{B}(\sigma)$. Hence $\mathrm{B}\left(\rho^{\alpha}\right)=\mathrm{B}(\rho) \alpha=\mathrm{B}(\sigma)$ for some $\alpha \in \Omega(V)$ by Lemma 9.2 and we get $\rho^{\alpha}=\sigma$. So $\rho \sigma=\rho \alpha^{-1} \rho^{-1} \alpha$ is a commutator in elements of $\Omega(V)$.

LEMMA 9.4. Let $K$ be a euclidean field and $\pi \in \Omega(V)$ be such that $V$ does not contain an orthogonally indecomposable $\pi$-module of type $2^{*}$. Then $\pi=\rho \sigma$ and $\mathrm{B}(\rho)$ is isometric to $\mathrm{B}(\sigma)$ for some orthogonal involutions $\rho, \sigma$.

PROOF. We will prove the assertion under each of the following additional assumptions and explain later why the assertion follows.

(i) $V$ is an orthogonally indecomposable $\pi$-module.

(ii) $V=U \oplus W$ where $\pi$-type $(U)=3=\pi$-type $(W)$.

(iii) $V=U \oplus W$ where $\pi-\operatorname{type}(U)=2^{+}=\pi-\operatorname{type}(W)$. 
(iv) $V=U \oplus W \oplus Z$ where $\pi-\operatorname{type}(U)=2^{+}=\pi-\operatorname{type}(W)$ and $\pi-\operatorname{type}(Z)=3$.

As $K$ is a euclidean field recall that

(1) Two regular subspaces of $\mathrm{V}$ are isometric if and only if they have the same signature.

An immediate consequence is

(2) If $A, B \leq V$ are regular subspaces and $m:=\operatorname{dim}(A)=\operatorname{dim}(B)$ is odd and ind $(A)=(m-1) / 2=\operatorname{ind}(B)$ and $\mathrm{d} A=\mathrm{d} B$ then $A$ is isometric to $B$.

(3) Let $\pi=\rho \sigma \in \operatorname{ker}(\Theta)$ where $\rho, \sigma$ are orthogonal involutions with $m:=$ $\operatorname{dim}(\mathrm{B}(\rho))=\operatorname{dim}(\mathrm{B}(\sigma))$. If $m$ is even and $\operatorname{ind}(\mathrm{B}(\rho))=m / 2=\operatorname{ind}(\mathrm{B}(\sigma))$, or if $m$ is odd and ind $(\mathrm{B}(\rho))=(m-1) / 2=\operatorname{ind}(\mathrm{B}(\sigma))$ then $\mathrm{B}(\rho)$ is isometric to $\mathrm{B}(\sigma)$.

Clearly, this follows from (2) since $\mathrm{dB}(\rho)=\Theta(\rho)=\Theta(\sigma)=\mathrm{dB}(\sigma)$.

Now we will prove the assertion in the special cases (i) to (iv).

(i) If $\pi$-type $(V)=2^{+}$then $\pi \in \mathrm{O}^{-}(V)$; hence this case does not occur. Furthermore, $2^{*}$ is excluded. If $\pi$-type $(V)=2^{-}$the assertion follows from (3) and Lemma 5.1. If $\pi$-type $(V)=1$ see Lemma 5.5 and (3). If $\pi$-type $(V)=3$ then the assertion follows from Lemma 5.7 and (3).

(ii) We may assume that $\Theta\left(\pi_{U}\right)=-K^{* 2}=\Theta\left(\pi_{W}\right)$ (or else the assertion follows from case (i)). Then Lemma 5.7 supplies $\rho_{1}, \sigma_{1} \in \mathrm{O}(U)$ and $\rho_{2}, \sigma_{2} \in \mathrm{O}(W)$ such that $\pi_{U}=\rho_{1} \sigma_{1}, \operatorname{sgn}\left(\mathrm{B}\left(\rho_{1}\right)\right)=(r, s), \operatorname{sgn}\left(\mathrm{B}\left(\sigma_{1}\right)\right)=(r-1, s)$ and $\pi_{W}=\rho_{2} \sigma_{2}$, $\operatorname{sgn}\left(\mathrm{B}\left(\rho_{1}\right)\right)=\left(r^{\prime}, s^{\prime}\right), \operatorname{sgn}\left(\mathrm{B}\left(\sigma_{1}\right)\right)=\left(r^{\prime}+1, s^{\prime}\right)$ for numbers $r, s, r^{\prime}, s^{\prime} \in \mathbb{N}_{0}$. Hence the identity $\pi=\left(\rho_{1} \oplus \rho_{2}\right)\left(\sigma_{1} \oplus \sigma_{2}\right)$ and (1) prove the assertion.

(iii) We have $(-\pi)$-type $(U)=2^{-}$, hence $\mathrm{d} U \cdot \Theta\left(\pi_{U}\right)=\Theta\left(-\pi_{U}\right)=K^{* 2}$. So $\Theta\left(\pi_{U}\right)=\mathrm{d} U$ and analogously $\Theta\left(\pi_{W}\right)=\mathrm{d} W$. As $\Theta(\pi)=K^{* 2}$ we conclude that $\mathrm{d} U=\mathrm{d} W=K^{* 2}$ or $\mathrm{d} U=\mathrm{d} W=-K^{* 2}$. We discuss the first case (the second one is similar). So let $\Theta\left(\pi_{U}\right)=\Theta\left(\pi_{W}\right)=K^{* 2}$. Using Lemma 5.1 and Corollary 5.10 we get orthogonal involutions $\rho_{i}, \sigma_{i}$ such that $\pi_{U}=\rho_{1} \sigma_{1}, \operatorname{sgn}\left(\mathbf{B}\left(\rho_{1}\right)\right)=(r, s)$, $\operatorname{sgn}\left(\mathrm{B}\left(\sigma_{1}\right)\right)=(r-1, s)$, and $\pi_{w}=\rho_{2} \sigma_{2}, \operatorname{sgn}\left(\mathrm{B}\left(\rho_{2}\right)\right)=\left(r^{\prime}, s^{\prime}\right), \operatorname{sgn}\left(\mathrm{B}\left(\sigma_{2}\right)\right)=$ $\left(r^{\prime}+1, s^{\prime}\right)$ where $r, s, r^{\prime}, s^{\prime} \in \mathbb{N}_{0}$. Hence $\pi=\left(\rho_{1} \oplus \rho_{2}\right)\left(\sigma_{1} \oplus \sigma_{2}\right)$ and $\mathrm{B}\left(\rho_{1} \oplus \rho_{2}\right)$ is isometric to $\mathrm{B}\left(\sigma_{1} \oplus \sigma_{2}\right)$.

(iv) One has $\pi_{Z} \in \mathrm{O}^{+}(Z)$. If $\Theta\left(\pi_{Z}\right)=K^{* 2}$ then $\pi_{Z} \in \Omega(Z)$ and $\pi_{U \Phi w} \in$ $\Omega(U \oplus W)$; hence (i) and (iii) yield the assertion. So let $\Theta\left(\pi_{Z}\right)=-K^{* 2}$. We may assume that $\Theta\left(\pi_{U}\right)=K^{* 2}$ and $\Theta\left(\pi_{W}\right)=-K^{* 2}$. Using 5.1, 5.10 and 5.7 we obtain orthogonal involutions $\rho_{i}, \sigma_{i}$ such that:

$$
\begin{aligned}
& \pi_{U}=\rho_{1} \sigma_{1}, \operatorname{sgn}\left(\mathrm{B}\left(\rho_{1}\right)\right)=(r, s), \operatorname{sgn}\left(\mathrm{B}\left(\sigma_{1}\right)\right)=(r+1, s) ; \\
& \pi_{W}=\rho_{2} \sigma_{2}, \operatorname{sgn}\left(\mathrm{B}\left(\rho_{2}\right)\right)=\left(r^{\prime}, s^{\prime}\right), \operatorname{sgn}\left(\mathrm{B}\left(\sigma_{2}\right)\right)=\left(r, s^{\prime}-1\right) ; \text { and } \\
& \pi_{Z}=\rho_{3} \sigma_{3}, \operatorname{sgn}\left(\mathrm{B}\left(\rho_{1}\right)\right)=\left(r^{\prime \prime}, s^{\prime \prime}\right), \operatorname{sgn}\left(\mathrm{B}\left(\sigma_{1}\right)\right)=\left(r^{\prime \prime}-1, s^{\prime \prime}+1\right)
\end{aligned}
$$

where $r, s, r^{\prime}, s^{\prime}, r^{\prime \prime}, s^{\prime \prime} \in \mathbb{N}_{0}$ are suitable numbers. Hence $\pi=\left(\rho_{1} \oplus \rho_{2} \oplus \rho_{3}\right)$ $\left(\sigma_{1} \oplus \sigma_{2} \oplus \sigma_{3}\right)$ and $\mathrm{B}\left(\rho_{1} \oplus \rho_{2} \oplus \rho_{3}\right)$ is isometric to $\mathrm{B}\left(\sigma_{1} \oplus \sigma_{2} \oplus \sigma_{3}\right)$. 
Why does the general assertion follow from the four special cases that have been dealt with ?

Suppose that the assertion is wrong. Then some $\pi \in \Omega(V)$ does nor satisfy the claim, and we can take an example where $\operatorname{dim}(V)$ is minimal. Clearly, $V$ does not admit a proper decomposition $V=A \oplus B$ into $\pi$-modules such that $\pi_{A} \in \Omega(A)$. In particular, $V$ is not an orthogonally indecomposable $\pi$-module (special case (i)), and a decomposition of $V$ into orthogonally indecomposable $\pi$-modules does not contain modules of type 1 or $2^{-}$or (by assumption) $2^{*}$. Hence only modules of type $2^{+}$and 3 can occur, and modules of $\pi$-type 3 have spinorial norm $-K^{* 2}$. Pairs of modules of $\pi$-type $2^{+}$with the same spinorial norm cannot occur (due to case (iii)), and the same statement holds true for type 3 (due to case (ii)). Hence at most one module of type 3 occurs (only type 3 modules with negative spinorial norm are admitted), at most one type $2^{+}$module with negative spinorial norm, and at most one type $2^{+}$module with positive spinorial norm. So we arrive at case (iv).

The proof is finished.

COROLLARY 9.5. Let $K=\mathbb{R}$ and $\pi \in \Omega(V)$. Then $\pi=\rho \sigma$ for involutions $\rho, \sigma \in \mathrm{O}(V)$ where $\mathrm{B}(\rho)$ and $\mathrm{B}(\sigma)$ are isometric.

PROOF. This follows immediately from the preceding lemma and Remark 5.4.

THEOREM 9.6. Let $\mathrm{u}(K) \leq 2$ or $K=\mathbb{B}$. Then $\mathrm{cl}(\mathrm{O}(V))=1$, that is, every element of $\Omega(V)$ is a commutator in elements of $\mathrm{O}(V)$.

PROOF. Let $\pi \in \Omega(V)$. First let $\mathbf{u}(K) \leq 2$. From Corollary 5.10 we obtain involutions $\rho, \sigma \in \mathrm{O}(V)$ such that $\pi=\rho \sigma$ and $\operatorname{dim}(\mathrm{B}(\rho))=\operatorname{dim}(\mathrm{B}(\sigma))$. Then $\mathrm{d} B(\rho)=\mathrm{d} B(\sigma)$ as $\Theta(\pi)=K^{* 2}$. Now Lemmas 2.3 and 9.2 yield some $\alpha \in \Omega(V)$ such that $\mathrm{B}\left(\rho^{\alpha}\right)=\mathrm{B}(\rho) \alpha=\mathrm{B}(\sigma)$ and we get $\rho^{\alpha}=\sigma$. So $\rho \sigma=\rho \alpha^{-1} \rho^{-1} \alpha$ is a commutator in elements of $\mathrm{O}(V)$.

If $K=\mathbb{R}$, the assertion follows from Corollary 9.5 and Lemma 9.2.

LEMMA 9.7. Let $n \geq 3$ and $\pi \in \Omega(V)$. Let $\rho, \sigma \in \mathrm{O}(V)$ be involutions with $\pi=\rho \sigma$ and suppose that (a) or (b) hold true:

(a) $\mathrm{u}(K) \leq 2$ and $[\rho, \sigma$ are symmetries and $n \geq 5]$ or $[\operatorname{dim}(\mathrm{B}(\rho))=2=$ $\operatorname{dim}(\mathrm{B}(\sigma))$ and $n \geq 8 \mathrm{]}$.

(b) $K=\mathbb{B}$, ind $(V) \neq 2, \operatorname{dim}(\mathrm{B}(\rho))=\operatorname{dim}(\mathrm{B}(\sigma)) \leq 2$ and $\operatorname{sgn}(\mathrm{B}(\rho))=$ $\operatorname{sgn}(\mathrm{B}(\sigma))$.

Then $\operatorname{cl}(\pi)=1$, that is, $\pi$ is a commutator in elements of $\Omega(V)$. 
PROOF. We may assume that $\pi \neq 1$. Choose a regular subspace $U \leq \mathrm{F}(\pi)$ of maximal dimension and let $W:=U^{\perp}$. Then $B(\pi)=\mathrm{F}(\pi)^{\perp} \leq W$. First suppose that (a) holds true.

Case 1: $\rho$ and $\sigma$ are symmetries and $n \geq 5$. Then $\operatorname{dim}(\mathrm{B}(\pi)) \leq 2$ and $\mathrm{B}(\pi)=$ $\mathrm{B}(\rho) \oplus \mathrm{B}(\sigma) \leq W$ is not totally isotropic. So $n>\operatorname{dim}(U) \geq n-3 \geq 2$. As $\mathrm{u}(K) \leq 2$ we find a symmetry $\omega$ such that $\mathrm{B}(\omega) \leq U$ and $\Theta(\omega)=\Theta(\rho)=\Theta(\sigma)$. Hence $\rho^{\prime}:=\rho \omega$ and $\sigma^{\prime}:=\sigma \omega \in \Omega(V)$ are 2-dimensional involutions such that $\pi=\rho^{\prime} \sigma^{\prime}$. The assertion follows from Corollary 9.3.

Case 2: $\operatorname{dim}(B(\rho))=\operatorname{dim}(B(\sigma))=2$. We may assume that $\pi$ is not a product of two symmetries (case 1) and that $\mathrm{B}(\rho), \mathrm{B}(\sigma) \leq W$; cf. Proposition 4.6. Furthermore, $\operatorname{dim}(B(\pi)) \leq 4$ and $\operatorname{dim}(\operatorname{rad}(B(\pi))) \leq 2$ (consider, for example, orthogonally indecomposable $\pi$-modules). Hence $\operatorname{dim}(U) \geq n-6 \geq 2$. If $\operatorname{dim}(U) \geq 3$ then we find a 2-dimensional subspace $T \leq U$ such that $\mathrm{d} T=\Theta(\rho)$. Let $\kappa$ denote the orthogonal involution with negative space $T$. Then $\pi=(\rho \kappa)(\sigma \kappa)$ is the product of two involutions in $\Omega(V)$ with 4-dimensional path and Corollary 9.3 yields the assertion. Let $\operatorname{dim}(U)=2$. Then $\operatorname{dim}(\mathrm{B}(\pi))=4$ and $\operatorname{dim}(\operatorname{rad}(\mathrm{B}(\pi)))=2$. Hence $W=X \oplus Y$ where $\pi-\operatorname{type}(X)=2^{-}=\pi-\operatorname{type}(Y)$ and $\operatorname{dim}(X)=3=\operatorname{dim}(Y)$. Now 5.1 and 5.2 supply $\pi=\eta \omega$ where $\eta$ and $\omega$ are orthogonal involutions such that $\mathrm{B}(\eta)$ and $\mathrm{B}(\omega)$ are 4-dimensional hyperbolic spaces. So 9.3 yields the assertion.

Now we prove part (b).

Case 1: $\operatorname{dim}(\mathrm{B}(\rho))=1$. As $\mathrm{B}(\pi)=\mathbf{B}(\rho) \oplus \mathbf{B}(\sigma) \leq W$ is not totally isotropic we find a 3-dimensional regular subspace $X$ such that $\mathrm{B}(\pi) \leq X$. If ind $(X)=1$ then Lemma 9.1 yields the assertion. Otherwise $X$ is anisotropic, and $X=\mathrm{B}(\pi) \oplus Y$ where $Y:=X \cap \mathrm{F}(\pi)$. Let $\kappa$ be the symmetry with negative space $Y$. Then $\pi=(\rho \kappa)(\sigma \kappa)$ is a product of two involutions in $\Omega(V)$ whose paths are isometric. The assertion follows from Corollary 9.3.

Case 2: $\operatorname{dim}(\mathrm{B}(\rho))=2$. We may assume that $\pi$ is not a product of two symmetries. So $\operatorname{dim}(W) \geq 4$. Due to 4.6 we may assume that $\mathrm{B}(\rho), \mathrm{B}(\sigma) \leq W$. We have $\operatorname{dim}(B(\pi)) \leq 4, \operatorname{dim}(\operatorname{rad}(B(\pi))) \leq 2$ and $4 \leq W \leq 6$.

If $\rho \in \Omega(V)$ then the assertion follows immediately from 9.3: Otherwise $\mathrm{B}(\rho)$ and $\mathrm{B}(\sigma)$ are hyperbolic planes. If $U$ is isotropic then we take a hyperbolic plane $H \leq U$ and have $\pi=(\rho \kappa)(\sigma \kappa)$, a product of two involutions in $\Omega(V)$ whose paths are isometric (4-dimensional hyperbolic spaces).

Suppose that $U$ is anisotropic. We discuss the case $\operatorname{sgn}(U)=(0, \operatorname{dim}(U))$, using 4.6, 5.1 and 5.2.

If $\operatorname{dim}(W)=6$ then $\operatorname{dim}(\mathrm{B}(\pi))=4$ and $\operatorname{dim}(\operatorname{rad}(\mathrm{B}(\pi)))=2$. This implies that $W=X \oplus Y$ where $X, Y$ are both 3-dimensional orthogonally indecomposable $\pi$-modules of type $2^{-}$. From 5.1 and 5.2 it follows that $\pi=\rho^{\prime} \sigma^{\prime}$ where $\rho^{\prime}, \sigma^{\prime}$ are 
orthogonal involutions whose paths are 4-dimensional hyperbolic spaces.

Now let $\operatorname{dim}(W)=5$. Then

(1) $W$ is an orthogonally indecomposable $\pi$-module of type $2^{-}$, or

(2) $W=X \oplus Y$ where $X, Y$ are $\pi$-modules, $\pi-\operatorname{type}(X)=2^{-}$and $\operatorname{dim}(X)=3$.

Consider the first possibility. Then ind $(W)=2$, and as ind $(V) \neq 2$ this yields that $\operatorname{sgn}(W)=(3,2)$ and $n \neq 5$. Thus 5.1 supplies orthogonal involutions $\rho^{\prime \prime}, \sigma^{\prime \prime}$ such that $\pi=\rho^{\prime \prime} \sigma^{\prime \prime}$ and $\operatorname{sgn}\left(\mathrm{B}\left(\rho^{\prime \prime}\right)\right)=(2,1)=\operatorname{sgn}\left(\mathrm{B}\left(\sigma^{\prime \prime}\right)\right)$, and we have $y \in U$ such that $q(y)=-1$. So $\pi=\rho^{\prime} \sigma^{\prime}$ where $\rho^{\prime}:=\rho^{\prime \prime} \sigma_{y}$ and $\sigma^{\prime}:=\sigma^{\prime \prime} \sigma_{y}$ proves the assertion ( $\sigma_{y}$ denotes the symmetry whose negative space is $\left.\langle y\rangle\right)$. Now we study the second possibility. We may assume that $Y$ is not a hyperbolic plane (otherwise 4.9 yields that $\pi$-type $(Y) \neq 2^{*}$; hence $\pi$-type $(Y)=3$ and using 5.8 we can prove the claim). As ind $(V) \neq 2$ one of the following situations occurs:

(a) $\operatorname{sgn}(Y)=(0,2)$ and $\operatorname{sgn}(X)=(1,2)$; or

(b) $\operatorname{sgn}(Y)=(2,0)$ and $\operatorname{sgn}(X)=(2,1)$; or

(c) $\operatorname{sgn}(Y)=(2,0)$ and $\operatorname{sgn}(X)=(1,2)$ and $n \neq 5$.

So $\pi_{Y}$ is in all three situations a product of two symmetries which are in cases (b) and (c) both positive, and in case (a) both negative. Using this remark and 5.1 (applied to $\pi_{X}$ ) we obtain $\pi=\rho^{\prime} \sigma^{\prime}$ for orthogonal involutions $\rho^{\prime}, \sigma^{\prime}$ such that in case (a) $\operatorname{sgn}\left(B\left(\rho^{\prime}\right)\right)=(0,2)=\operatorname{sgn}\left(B\left(\sigma^{\prime}\right)\right)$, in case $(\mathrm{b}) \operatorname{sgn}\left(B\left(\rho^{\prime}\right)=(2,0)=\operatorname{sgn}\left(B\left(\sigma^{\prime}\right)\right)\right.$, and in case (c) $\operatorname{sgn}\left(B\left(\rho^{\prime}\right)=(2,1)=\operatorname{sgn}\left(B\left(\sigma^{\prime}\right)\right)\right.$. In cases (a) and (b) we have finished, as $\rho^{\prime}, \sigma^{\prime} \in \Omega(V)$ and Corollary 9.3 applies. In case (c) we take a symmetry $\kappa$ such that $\mathrm{B}(\kappa) \leq U$. Then $\kappa$ is negative and $\rho^{\prime \prime}:=\rho^{\prime} \kappa, \sigma^{\prime \prime}:=\sigma^{\prime} \kappa$ fulfil the requirements. Again 9.3 yields the assertion.

Finally, we study the case $\operatorname{dim}(W)=4$. Then $\operatorname{ind}(W) \neq 2$ as $\operatorname{ind}(V) \neq 2$. Furthermore, $W$ is isotropic as $B(\rho)$ is isotropic and $\mathrm{B}(\rho) \subseteq W$. Hence ind $(W)=1$ and $\mathrm{d} W=-K^{* 2}$. Therefore, $-\rho_{W}=-1_{W} \cdot \rho_{W} \in \Omega(W)$. The same argument applies to $\sigma$. Thus $\pi=\left(-\rho_{W}(1) 1_{U}\right)\left(-\sigma_{W} \oplus 11_{U}\right)$ proves the assertion.

THEOREM 9.8. Let $n \geq 3$ and let $\mathbf{u}(K) \leq 2$ or $[K=\mathbb{R}$ and ind $(V) \neq 2]$. If $|K|=3$ suppose additionally that $n \geq 5$ or $[n=4$ and $\operatorname{ind}(V)=1]$. Then $\operatorname{cl}(\Omega(V)) \leq 2$.

ProOF. Let $\pi \in \Omega(V) \backslash\{1\}$.

First suppose that $\mathrm{u}(K) \leq 2$. If $n \in\{3,4\}$ and $\operatorname{ind}(V)=1$ then 9.1 shows that $\operatorname{cl}(\Omega V))=1$. If $n=4$ and ind $(V)=2$ then $\operatorname{cl}(\operatorname{PS} \Omega(V))=1$; this follows from 4.2 and $\operatorname{cl}\left(\mathrm{PSL}_{2}(K)=1 ; \operatorname{cf}\right.$. [17]. Hence $\operatorname{cl}(\pi)=1$ or $\operatorname{cl}(-\pi)=1$. One has a decomposition $V=A \oplus B$ where $A, B$ are 2-dimensional subspaces such that $\mathrm{d} A=\mathrm{d} B=K^{* 2}$. Let $\rho$ and $\sigma$ denote the orthogonal involutions with negative spaces $A$ respectively $B$. Then $-1=\rho \sigma$; hence $\operatorname{cl}(-1)=1$ by 9.3 . So $\operatorname{cl}(\pi) \leq 2$. 
Now we assume that $n \geq 5$. Take involutions $\rho, \sigma \in \mathrm{O}(V)$ such that $\pi=\rho \sigma$ and $k:=\operatorname{dim}(\mathrm{B}(\rho))=\operatorname{dim}(\mathrm{B}(\sigma))$ and $k$ is odd unless $n \equiv 0 \bmod 4$; cf. Corollary 5.10 .

Case 1: $k$ is odd. As $\mathbf{u}(K) \leq 2$ we find symmetries $\omega$ and $\varphi$ such that $\mathbf{B}(\omega) \leq \mathbf{B}(\rho)$, $\mathrm{B}(\varphi) \leq \mathrm{B}(\sigma)$ and $\Theta(\omega)=\Theta(\rho)=\Theta(\varphi)$. Then $\pi=\rho \sigma=\omega \varphi(\omega \rho)^{\varphi}(\varphi \sigma)$. Clearly, $(\omega \rho)^{\varphi}$ and $\varphi \sigma$ are involutions in $\Omega(V)$ and their negative spaces have the same dimension. Hence $\operatorname{cl}\left((\omega \rho)^{\varphi} \varphi \sigma\right)=1$ by 9.3 . Furthermore, $\operatorname{cl}(\omega \varphi)=1$ by 9.7 (a). So $\mathrm{cl}(\pi) \leq 2$.

Case 2: $k$ is even. Then $n \geq 8$ by our choice of $k$. As u $(K) \leq 2$ we find orthogonal involutions $\omega$ and $\varphi$ with 2-dimensional negative spaces such that $\mathrm{B}(\omega) \leq \mathrm{B}(\rho)$, $\mathrm{B}(\varphi) \leq \mathrm{B}(\sigma)$ and $\Theta(\omega)=\Theta(\rho)=\Theta(\varphi)$. Now apply the same arguments as in the first case.

Second, let $K=\mathbb{R}$ and ind $(V) \neq 2$. Then Corollary 9.5 supplies involutions $\rho, \sigma \in$ $\mathrm{O}(V)$ such that $\pi=\rho \sigma$ and $\operatorname{sgn}(\mathrm{B}(\rho))=\operatorname{sgn}(\mathrm{B}(\sigma))$. Thus we find involutions $\kappa, \eta \in$ $\mathrm{O}(V)$ such that $\mathrm{B}(\kappa) \leq \mathrm{B}(\rho), \mathrm{B}(\eta) \leq \mathrm{B}(\sigma), \operatorname{sgn}(\mathrm{B}(\kappa))=\operatorname{sgn}(\mathrm{B}(\eta)), \kappa \rho, \eta \sigma \in \Omega(V)$ and $\operatorname{dim}(\mathrm{B}(\kappa))=\operatorname{dim}(\mathrm{B}(\eta)) \leq 2$. As $(\kappa \rho)^{\eta}$ and $\eta \sigma$ are involutions in $\Omega(V)$ whose paths have the same signatures their product is a commutator in elements of $\Omega(V)$; cf. 9.3. Also $\kappa \eta$ is a commutator in elements of $\Omega(V)$; cf. 9.7.

(b). As $\pi=\kappa \eta(\kappa \rho)^{\eta}(\eta \sigma)$ we conclude that $\operatorname{cl}(\pi) \leq 2$. The proof is finished.

Under the additional assumption that $V$ is anisotropic the following theorem was already proved in [19].

THEOREM 9.9. Let $n \geq 3, K=\mathbb{R}$ and $m:=\operatorname{ind}(V) \leq 1$. Then $\operatorname{cl}(\Omega(V))=1$, that is, every element of $\Omega(V)$ is a commutator in elements of $\Omega(V)$.

PROOF. If $n=3$ and $m:=\operatorname{ind}(V)=1$ then Lemma 9.1 yields the assertion. Let $n \geq 3+m$. We may assume that $\operatorname{sgn}(V)=(n-m, m)$. Let $\pi \in \Omega(V)$.

We claim:

(*) $V$ admits an orthogonal decomposition into $\pi$-modules $W$ such that each $W$ has one of the following four forms

(i) $\operatorname{dim}(W)=1$ and $\pi_{W}=1_{W}$.

(ii) $W$ is a hyperbolic plane and $\pi_{W}=\left(\begin{array}{cc}\lambda & 0 \\ 0 & \lambda-1\end{array}\right)$ where $\lambda \in \mathbb{R}_{>0}$ and $\lambda \notin\{1,-1\}$.

(iii) W is anisotropic and $\pi_{W}=\left(\begin{array}{cc}\cos (\alpha) & \sin (\alpha) \\ -\sin (\alpha) & \cos (\alpha)\end{array}\right)$

(iv) $\operatorname{dim}(W)=3, \mathrm{~d} W=-K^{* 2}$ and $\pi-\operatorname{type}(W)=2^{-}$(that is, $\pi_{W}$ is an Eichlertransformation whose path is not totally isotropic).

Furthermore, we claim that $\pi_{W} \in \Omega(W)$ for each summand $W$ which does not have form (i). 
PROOF OF $(*)$. Consider an orthogonal decomposition of $V$ into orthogonally indecomposable $\pi$-modules. Let $U$ be such a $\pi$-module. As $m \leq 1$ it follows that $\pi-\operatorname{type}(U) \neq 1$, and

(a) if $\pi-\operatorname{type}(U)=3$ then $\operatorname{dim}(U)=2$,

(b) if $\pi-\operatorname{type}(U)=2^{+}$or $2^{-}$then $\operatorname{dim}(U) \leq 3$,

(c) if $\pi$-type $(U)=2^{*}$ then $\operatorname{mip}\left(\pi_{U}\right)=p^{t}$ where $p \in \mathbb{R}[x]$ is irreducible of degree 2; as $m \leq 1$ it follows that $t=1$, hence $\operatorname{dim}(U)=2$ (cf. Lemma 2.10).

So $\operatorname{dim}(U) \leq 3$ in each case, and if $U$ is anisotropic then $\operatorname{dim}(U) \leq 2$. Furthermore, $\Theta\left(\pi_{U}\right)=K^{* 2}$ for each $U$ (if $\Theta\left(\pi_{U}\right)=-K^{* 2}$ then $\pi_{U} \neq 1_{U}$ and $U$ contains a vector u such that $\mathrm{f}(u, u)<0$. As $\operatorname{sgn}(V)=(n-1,1)$ this implies that $\Theta\left(\pi_{Z}\right)=K^{* 2}$ for each $\pi$-module $Z \neq U$ of the decomposition. Hence we get the contradiction $\Theta(\pi)=-K^{* 2}$ ). In particular, a 3-dimensional $U$ where $\pi$-type $(U)=2^{+}$does not occur; cf. 4.7 (d). Hence, if $\operatorname{det}\left(\pi_{U}\right)=-1$, then $\pi-\operatorname{type}(U)=2^{+}$and $\operatorname{dim}(U)=1$, that is, $\pi_{U}$ is a symmetry. We can put these 1-dimensional $\pi$-modules together to pairs $\mathrm{W}$ as in (iii) (with $\cos (\alpha)=-1$ ). The previous statements prove $\left({ }^{*}\right)$ and the 'furthermore' statement too.

Take an orthogonal decomposition into $\pi$-modules $W$ of forms (i) to (iv).

Obviously we may assume: At most one module of the form (i) occurs. For each $\pi$-module $W$ of the form (ii), (iii) or (iv) we claim

(a) $\pi_{W}=\rho \sigma$ for symmetries $\rho, \sigma \in \mathrm{O}(W)$ such that $\Theta(\rho)=K^{* 2}=\Theta(\sigma)$, and

(b) $\pi_{W}=\psi^{2}$ and $\psi^{\omega}=\psi^{-1}$ for a symmetry $\omega \in O(W)$ and some $\psi \in \Omega(W)$.

PROOF OF (a). In case (ii) this follows from Lemma 5.7; in case (iii) it follows from Scherk's theorem (Proposition 4.12) and from $\operatorname{sgn}(W)=(2,0)$; in case (iv) apply 5.1 and 5.2.

PROOF OF (b). We will find $\psi \in \Omega(V)$ such that $\psi^{2}=\pi_{W}$ and $\psi$ is also of type (ii), (iii) or (iv). Then we write $\psi$ in the form given by (a) (with $\pi_{w}$ replaced by $\psi$ ) and thus obtain $\omega$ as in (b).

In case (ii) take $\psi=\left(\begin{array}{ll}\mu & \\ 0 & \mu^{-1}\end{array}\right)$ where $\mu$ is the square root of $\lambda$.

In case (iii) take $\psi=\left(\begin{array}{cc}\cos (\beta) & \sin (\beta) \\ -\sin (\beta) & \cos (\beta)\end{array}\right)$ where $\beta:=\alpha / 2$. In case (iv) one has an Eichler-transformation $\psi$ such that $\psi^{2}=\phi_{W}(K=\mathbb{R}$ is not needed); cf. for example [3, p. 214].

We have proved (a) and (b).

$\left.{ }^{* *}\right)$ The assertion that $\pi$ is a commutator in elements of $\Omega(V)$ holds true whenever 
$V=A \oplus B$ or $V=A \oplus B \oplus C$, where $A, B, C$ are $\pi$-modules $W$ of the form (i) to (iv) and at most one of them has form (i).

PROOF. In the first case we put together two by two symmetries supplied by (a) and get $\pi=\rho \sigma$ where $\rho, \sigma \in \Omega(V)$ are involutions such that $\operatorname{sgn}(\mathrm{B}(\rho))=(2,0)=$ $\operatorname{sgn}(\mathrm{B}(\sigma))$; hence $\pi$ is a commutator in elements of $\Omega(V)$; cf. 9.3 or 9.7 .

Now consider the second case. We may assume that $\operatorname{dim}(C)=2$ and have $\operatorname{dim}(A \oplus B) \geq 3$. The previous case yields involutions $\rho, \sigma \in \mathrm{O}(A \oplus B)$ such that $\operatorname{sgn}(B(\rho))=(2,0)=\operatorname{sgn}(B(\sigma))$ and $\pi_{A \oplus B}=\rho \sigma$. Furthermore, 9.2 supplies $\alpha^{\prime} \in \Omega(A \oplus B)$ such that $\sigma=\rho^{\alpha^{\prime}}$. Take a symmetry $\kappa$ such that $\mathrm{B}(\kappa) \leq \mathrm{B}(\rho)$ and let $\alpha:=\kappa \alpha^{\prime}$. Then $\sigma=\rho^{\alpha}$ and $\alpha \in \mathrm{O}^{-}(A \oplus B)$ and $\Theta(\alpha)=K^{* 2}$. Now (b) yields $\psi \in \mathrm{O}(C)$ such that $\pi_{C}=\psi^{2}$ and a symmetry $\omega \in \mathrm{O}^{-}(C)$ such that $\psi^{-1}=\psi^{\omega}$ and $\Theta(\omega)=K^{* 2}$. Thus $\pi=(\rho \oplus \psi)(\sigma \oplus \psi)=(\rho \oplus \psi)\left(\rho \oplus \psi^{-1}\right)^{(\alpha \oplus \omega)}$. Hence $\pi$ is a commutator in elements of $\Omega(V)$.

We proved have $\left({ }^{* *}\right)$.

Finally, as $n \geq 3$ and $n \geq 4$ in the isotropic case a decomposition given by $\left({ }^{*}\right)$ contains at least 2 modules $W$. Hence the assertion follows from (**).

\section{References}

[1] F. Bünger, F. Knüppel and K. Nielsen, 'Products of symmetries in unitary groups', Linear Algebra Appl. 260 (1997), 9-42.

[2] A. J. Hahn, 'The elements of the orthogonal group $\omega_{n}(v)$ as products of commutators of symmetries', Manuscript, Notre Dame, September 1994.

[3] J. Hahn and O. T. O'Meara, The classical groups and K-theory (Springer, Berlin, 1989).

[4] B. Huppert, 'Isometrien von Vektorräumen I', Arch. Math. 35 (1980), 164-176.

[5] —_, 'Isometrien von Vektorräumen II', Math. Z 175 (1980), 5-20.

[6] - Angewandte lineare Algebra (de Gruyter, Berlin, 1990).

[7] N. Ito, 'A theorem on the alternating group $A_{n}$ ( $n \geq 5$ )', Math. Japon. 2 (1951), 59-60.

[8] F. Knüppel, 'Products of involutions in orthogonal groups', Ann. Discrete Math. 37 (1988), 231248.

[9] — 'Products of simple isometries of given conjugacy types', Forum Math. 5 (1993), 441-458.

[10] F. Knüppel and K. Nielsen, 'On products of two involutions in the orthogonal group of a vector space', Linear Algebra Appl. 94 (1987), 209-216.

[11] _. 'SL(V) is 4-reflectional', Geom. Dedicata 38 (1991), 301-308.

[12] F. Lorenz, Quadratische Formen über Körpern, Lecture Notes in Math. 130 (Springer, Berlin, 1970).

[13] K. Nielsen, 'Conjugacy classes and commutators in symplectic groups', to appear.

[14] O. Ore, 'Some remarks on commutators', Proc. Amer. Math. Soc. 2 (1951), 307-314.

[15] J.-M. Quin, 'On commutators in orthogonal groups', Chinese J. Math. 5 (1965), 437-449. 
[16] P. Scherk, 'On the decomposition of orthogonalities into symmetries', Proc. Amer. Math. Soc. 1 (1950), 481-491.

[17] R. C. Thompson, 'Commutators in the special and general linear groups', Trans. Amer. Math. Soc. 1 (1950), 481-491.

[18] G. Thomsen, Läangenprobleme in klassischen Gruppen. Teil I: Involutionen mit zwei-dimensionalem Negativraum als Erzeugende der speziellen linearen Gruppe. Teil Il: Die Kommutatorgruppe der orthogonalen Gruppe und der Kern der Spinornorm (Dissertation, Kiel, 1991).

[19] H. Tôyama, 'On commutators of matrices', Kodai Math. Sem. Rep. 5-6 (1949), 1-2.

[20] G. E. Wall, 'On the conjugacy classes in the unitary, symplectic and orthogonal groups', J. Austral. Math. Soc. 3 (1963), 1-62.

[21] M. J. Wonenburger, 'Transformations which are products of two involutions', J. Math. Mech. 16 (1966), 327-338.

[22] H. Zassenhaus, 'On the spinor norm', Arch. Math. 13 (1962), 434-451.

\section{Mathematisches Seminar \\ Ludewig-Meyn-Straße 4 \\ D-24098 Kiel}

Germany 